\title{
Kinetics of pressure induced structural phase transitions-A review
}

\author{
N V CHANDRA SHEKAR* and K GOVINDA RAJAN \\ Materials Science Division, Indira Gandhi Centre for Atomic Research, Kalpakkam 603 102, India
}

MS received 20 October 2000

\begin{abstract}
The current status of experimental as well as theoretical advances in the understanding of kinetics of structural phase transitions is reviewed. A brief outline of the classification of phase transitions and classical ideas in the theory of kinetics of phase change is presented first. High pressure experimental techniques developed for studying the kinetics of structural transitions are reviewed and the salient features of each technique is brought out. The experimental technique using the diamond anvil cell (DAC) and image processing gets special mention as it promises to impart a new direction to this field. The usefulness of kinetic parameters in understanding the mechanism of a phase transition is examined. Typical examples from the literature are provided to give a flavour for these kind of studies. In conclusion, several open questions are raised which could pave way for future work in this area.
\end{abstract}

Keywords. High pressure; structural transitions; kinetics.

\section{Introduction}

In this paper, the present status of research on kinetics of structural phase transitions, both experimental and theoretical is reviewed. The cross-disciplinary subject of kinetics of phase transitions is of vital interest to condensed matter physicists. This is not only of academic importance, but also of technological relevance. The literature abounds in experimental studies and, to a lesser extent, in theoretical studies, and the subject is continually growing. Newer topics like amorphization under pressure have rejuvenated the interest in the subject.

This report is written with the perspective of a high pressure physicist and with particular interest in polymorphic transitions under high pressure. For the investigation of the thermodynamic and electronic equilibrium properties, a broad spectrum of methods for pressure generation and physical measurements have been developed (Jayaraman 1983, 1986; Hemley et al 1987; Spain and Paauwe 1977; Hochheimer and Etters 1991; Singh 1992). The measurement of equilibrium properties, however, gives only incomplete insight into the detailed mechanics of phase changes. The accurate determination of the phase equilibria and phase diagrams becomes harder at low temperatures due to increasing hysteresis effects. Consequently, the extrapolation of the experimental data to zero temperatures and the comparison with quantum mechanical $a b$ initio calculations become questionable. The study of phase transformation kinetics yields data on the reaction velocity and additional information on the basic mechanisms of the transition. This allows a more accurate extrapolation of the phase equilibria to low tem-

*Author for correspondence peratures (Merkau and Holzapfel 1986; Jeanloz 1987; Kruger et al 1990) and the comparison with theoretical results. In addition, the kinetics of phase changes under pressure is of basic importance for understanding the physical and chemical reactions that control the formation of high-pressure phases with special properties. Therefore a knowledge of the mechanisms of phase transformations is a presupposition for understanding the synthesis of new materials (Lorenz et al 1988). In geological applications, various crystallization processes cannot be studied under laboratory conditions because of long duration of the experiments. The information obtained from natural samples (e.g. igneous rocks) is restricted to the chemical, physical and structural analysis of the end product of series of different kinetic processes, which led to formation of the sample. The application of a kinetic model of phase transition can be useful to investigate more precisely the thermodynamic conditions of rock formation. However, the kinetics of the pressure induced transformations have not been studied as extensively as the kinetics of the temperature induced transformations mainly because of the difficulties in high pressure experiments.

The pressure induced transformations, which have been studied for their kinetic aspects fall under two categories. In the first category are the polymorphic transformations, which are displacive in nature. The transformations usually have low activation energies and are relatively rapid. Since polymorphic transformations can occur at relatively lower temperatures (room temperature or lower), any diffusion process is unlikely to take place. The second category of the transformations is the reconstructive type. These have large activation energy and are generally sluggish. The transformation rates become appreciable only at higher temperatures. Such transformations are often diffusion controlled. 
In $\S 2$, various classification schemes of phase transitions are described. The importance of structural classification schemes and their present level of understanding are briefly discussed. A brief introduction to the world of pressure induced structural phase transitions is given in $\S 3$.

Section 4 is devoted for reviewing the existing understanding on the kinetics of phase transitions from thermodynamics point of view. In addition, some basic and well known theoretical concepts relevant to the subject are reproduced. Various experimental techniques used for studying kinetics are described in $\S 5$. This also includes a section on kinetic studies in a diamond anvil cell (DAC). In $\S 6$, some interesting studies on kinetics of phase transformation, from the literature, are described. The conclusions and the future directions are laid out in $\S 7$.

\section{Classifications of phase transitions}

This brief section on classification scheme is included to review the status of kinetics as a parameter for classifying phase transitions. The importance and utility of other schemes are discussed for completion sake. Much of the earlier work on 'classifying' the changes from one structure to another was based on the rate with which the transition was effected. While crystallographic differences were recognized, a scheme for the classification of the transitions based on the nature of the crystallographic change was in fact the last to be developed. In between these two developments, the well known work of Ehrenfest provided a rigorous treatment of the thermodynamics to the case of transition between two solid phases. Roy (1973) has reviewed these classifications systematically. He has identified four principal approaches to the problem by classifying phase transitions based on kinetic, thermodynamic classification, thermochemical, and structural considerations.

The kinetic classification starts from the early work of Le Chatelier and simply divides transitions into two main groups viz. (i) rapid, or non-quenchable, and (ii) sluggish. However, no real classification scheme can be based on this parameter since it does not treat any basic property of either of the two phases. Moreover, as experimental capabilities grow, what is regarded as 'non quenchable' today may be established to be quenchable tomorrow. However, some recent research on this aspect has pointed out that with the right combination of the structural as well as the kinetic aspect an acceptable criterion of classification may emerge.

The thermodynamic classification leads to a very satisfactory conceptual scheme, dividing all phase transitions according to their 'order' and is summarized by the statements of table 1 . In actual practice, several corollary relations, which are demanded by these elementary relations, are frequently ignored.
Thermochemical classification proposes to classify all transitions by the magnitudes of $\Delta H$ (change in enthalpy) and $\Delta S$ (change in entropy). It was hoped that if a matrix of these two elements is constructed, transitions could be pigeonholed in it. The difficulty arises because of the unavailability/unreliability of the data on $\Delta H$ and $\Delta V$ etc and the extreme rarity of data on $\Delta \alpha$ (coefficient of thermal expansion) and $\Delta K$ (isothermal compressibility). The applicability of this scheme is limited because it does not treat the fundamental differences between the phases.

While the thermodynamic treatment of phase transitions is fundamental and useful, it does not provide a geometrical picture of the microscopic changes accompanying a transition. In fact, the major strides made in the understanding of the crystal chemistry (Rao and Rao 1967, 1978) in terms of atomic arrangements and bonding can be advantageously applied to the study of phase transitions in solids. For example, the new phase obtained after a transition may be related to the parent phase. The transition may have been accompanied by a change in the primary coordination or secondary coordination either being brought out by a reconstructive or displacive mechanism. The transition could be accompanied by a major change in the electronic structure or the bond type. A detailed study of the structures of the parent phase and the transformed phases, particularly looking for the presence of orientational relations between them, becomes important in understanding the mechanism of transition. It is now known that the transition in many solids may involve simple deformational (or orientational) relation between the two phases similar to those in the martensitic transitions common on metal systems. Recently, Gupta and Chidambaram (1994) have studied the symmetry systematics of the pressure induced structural phase transitions and proposed a classification into four categories: iso-symmetric, group-subgroup, intersection group and order-disorder transitions.

Table 1. Thermodynamic classification.

Classification by stability

Enantiotropic $=$ Reversible, proceeds in both directions (hence equilibrium attainable)

Monotropic $=$ Irreversible, proceeds in only one direction (no equilibrium possible between phases)

Equilibrium $=($ only requires reversibility $)$

Metastable $=$ Between two phases neither of which is the lowest $G$ phase

Stable $=$ Between two lowest free energy phase

\section{Classification by 'order'(after Ehrenfest)}

1 st order $=$ Discontinuity in 1 st derivative functions of $G$ 2nd order $=$ Discontinuity in 2nd derivative of $G$. Change of slope only in 1 st derivative property

$n$th order $=$ Discontinuity in the $n$th derivative property of $G$. Change of slope only in $(n-1)$ th derivative. 
A test on the utility of any classification is to see whether it enables us to understand a transition being studied. Figure 1 gives a three dimensional plot where it may be easy to pin point any type of transition. Table 2 gives the same in tabular form with many examples.

\section{Pressure induced phase transitions}

Pressure as a thermodynamic variable provides a means of changing the interatomic distances in materials in a controlled manner. Experimenters can now change the densities of condensed matter by upward of an entire order of

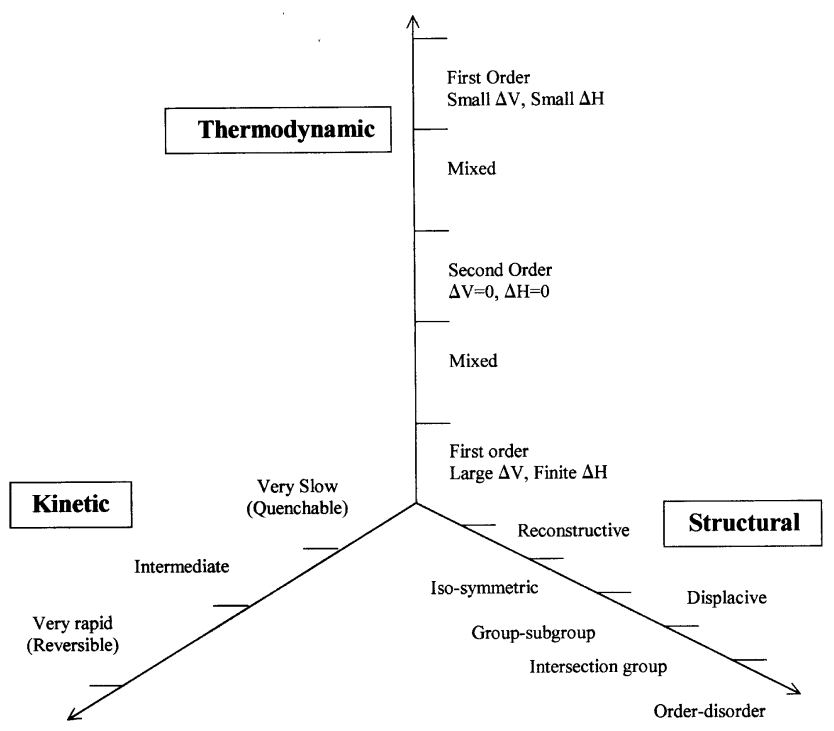

Figure 1. Classification scheme on a three-dimensional plot. magnitude, and thereby impart dramatic changes in physical and chemical properties of materials (Hemley and Mao 1997; Hemley and Ashcroft 1998). The decrease in the volume brought about by pressure can be more than $50 \%$ compared with only a few per cent change resulting from temperature increase in materials up to their melting point. Application of pressure has thus led to the discovery of phenomenon like the solid-solid critical point, molecular dissociation, new structural phases, etc (Klement and Jayaraman 1967; Pistorius 1976; Yousuf 1985; Jayaraman 1990; Ruoff 1991; Sahu 1994; Shekar 1997). Pressure induced phase transition in materials is more of a rule than an exception (Gupta and Chidambaram 1994).

During the past decade, there has been a great deal of development in the area of pressure induced phase transitions, both in the experimental techniques and theoretical methods. In the static high pressure experiments, the advances in the diamond anvil cell (DAC) technology and $\mathrm{X}$-ray diffraction techniques with the use of synchrotron radiation sources have facilitated detection of new phase transitions in solids under megabar pressures (Jayaraman 1983, 1986; Vohra et al 1986; Mao et al 1990; Yousuf 1998).

Also, in shock wave experiments it has become possible to detect phase transitions that are accompanied by small volume changes $(0.5 \%)$ by using a new technique based on the observation of a discontinuity in the measured sound velocity as a function of the peak pressure in the shocked state (Brown and McQueen 1986; Hixon et al 1989).

On the theoretical side, there are various approaches to analyse the pressure induced phase transitions. These vary from the use of the empirical methods based on the

Table 2. A schematic approach to describing a particular phase transition using observations in all 'dimensions' (Roy 1973).

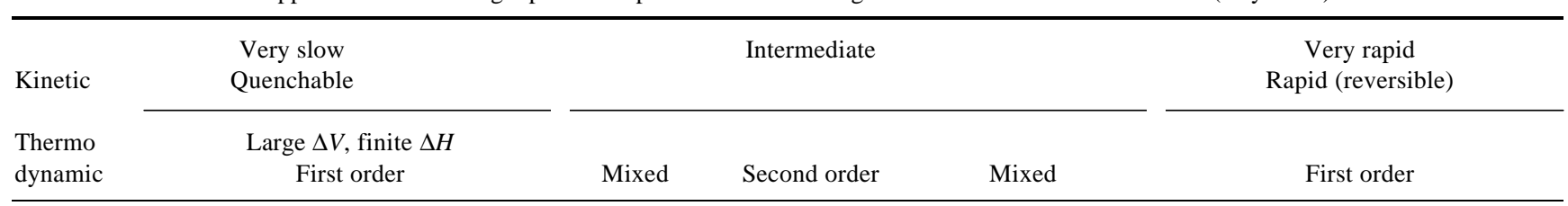

Structural relationships between parent and daughter phases:

None

Reconstructive

Structural

\begin{tabular}{|c|c|c|c|c|c|c|c|c|}
\hline & & & & & & & & \\
\hline & $\begin{array}{l}\text { Change in primary } \\
\text { coordination }\end{array}$ & $\begin{array}{l}\text { Change in } \\
\text { secondary } \\
\text { coordination }\end{array}$ & $\begin{array}{l}\text { Stacking } \\
\text { substitutional } \\
\text { rotational }\end{array}$ & & & $\begin{array}{l}\text { Iso- } \\
\text { symmetric }\end{array}$ & $\begin{array}{l}\text { Group- } \\
\text { subgroup }\end{array}$ & $\begin{array}{l}\text { Intersection } \\
\text { group }\end{array}$ \\
\hline Examples & $\begin{array}{l}\mathrm{C}_{\text {diamond }} \leftrightarrow \text { graphite } \\
\mathrm{SiO}_{2}, \text { qtz } \leftrightarrow \text { rut } \\
\mathrm{CaCO}_{3}, \mathrm{Calc} \leftrightarrow \text { arag } \\
\mathrm{CdS}, \mathrm{ZnS} \leftrightarrow \mathrm{NaCl}\end{array}$ & $\begin{array}{l}\mathrm{SiO}_{2}, \\
\mathrm{qtz} \leftrightarrow \text { trid } \\
\mathrm{Mg}_{2} \mathrm{SiO}_{4}, \\
\text { Oliv } \leftrightarrow \text { Spin }\end{array}$ & $\begin{array}{l}\mathrm{ZnS}, \\
3 \mathrm{C} \leftrightarrow 2 \mathrm{H} \\
\mathrm{Na}\left(\mathrm{AlSi}_{3}\right) \mathrm{O}_{5}, \\
\text { hi } \leftrightarrow \text { low }\end{array}$ & $\begin{array}{l}\mathrm{AuCu}_{3}, \\
\text { ord } \leftrightarrow \text { dis } \\
\mathrm{Ni}, \\
\text { ferro } \leftrightarrow \text { para }\end{array}$ & $\begin{array}{l}\text { Quartz, } \alpha \leftrightarrow \beta \\
\mathrm{BaTiO}_{3} \text {, tet } \leftrightarrow \mathrm{c} \\
\mathrm{Fe}_{3} \mathrm{O}_{4} \text {, rho } \leftrightarrow \mathrm{c} \\
\mathrm{V}_{2} \mathrm{O}_{3}, \text { mono } \leftrightarrow \mathrm{rh}\end{array}$ & $\begin{array}{l}\text { Recorsinol } \\
\alpha \leftrightarrow \beta\end{array}$ & $\begin{array}{l}\text { Th } \\
\text { fcc } \leftrightarrow \text { bct }\end{array}$ & $\begin{array}{l}\mathrm{Zr} \\
\omega \leftrightarrow \beta\end{array}$ \\
\hline
\end{tabular}

Close and space groups strictly governed by relationships

Order $\quad$ Ferro $\leftrightarrow$ paraelectric

disorder $\quad$ Ferro $\leftrightarrow$ paramagnetic

Metal $\leftrightarrow$ semiconductor

Conductor $\leftrightarrow$ super-
conductor 
general trends, to the application of the first principle calculations. With the increase in the computational speed and improvement in the theory, the ab initio band structure methods have acquired the capability to calculate small structural energy differences between different phases (a few milliRyd atom or smaller), so that successful prediction of the structural phase transitions is presently possible (Skriver 1985; Gupta 1992; Gupta et al 1993). Molecular dynamics methods are also becoming increasingly popular for analysing the mechanisms of phase transitions (Tse and Khy 1991; Chaplot and Sikka 1992, 1993).

Under pressure, most of the observed phase transitions are thought to be diffusionless in nature as pressure tends to suppress the large scale diffusion of atoms in the solid state. This is because compression very sharply increases the repulsive forces between neighbouring atoms, with the result that the height of the potential barrier, which must be overcome by the atoms in order to diffuse increases. So the continued increase in pressure gradually distorts the structure, molecular shapes and orientations and prepares the structure for undergoing phase transition with very little final displacement of the atoms. These small terminal displacements are usually reversible (even in crystalline to amorphous transitions) and maintain atomby-atom correspondence between the positions in the parent and the product structures. Because of this correspondence, the parent and the product phases display interesting symmetry relationships and the phase transitions can be analysed using Landau theory.

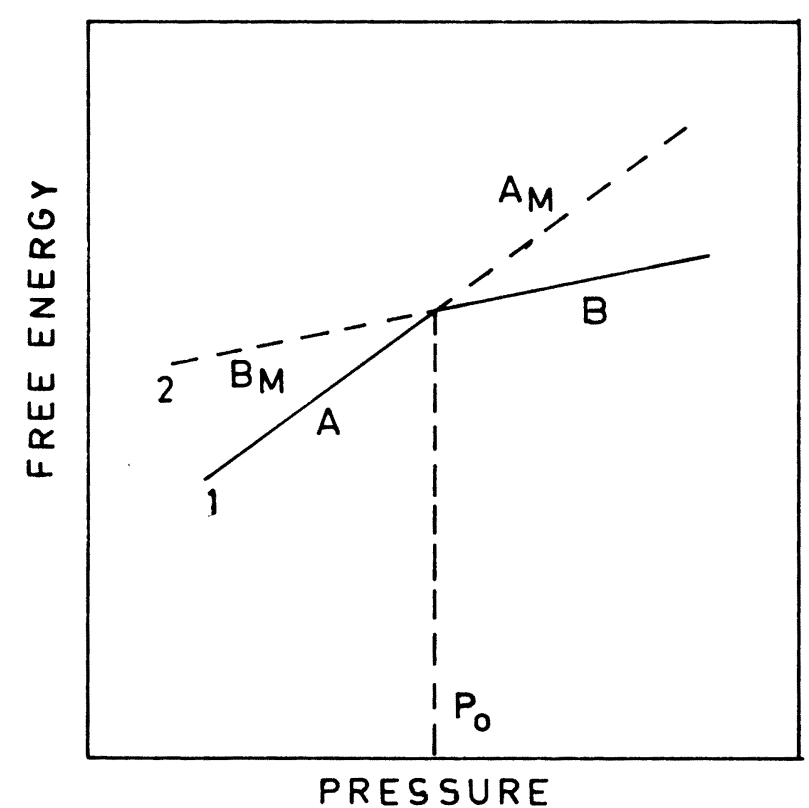

Figure 2. Variation of Gibbs free energy with pressure of phases $A$ and $B$ in the vicinity of $P_{0}$. Phase $A$ undergoes a first order transformation to phase $B$ above $P_{0}$.

\section{Thermodynamic considerations of kinetics of phase transitions}

The relative stabilities of the various phases (of the same chemical composition) can be conveniently discussed with reference to a diagram showing the variation of the Gibbs free energy, $G$, as a function of the thermodynamic variable. Such a diagram for a system which exhibits two phases $A$ and $B$ as the pressure is varied is shown in figure 2 . The lines marked 1 and 2 depict the variation of $G$ with pressure for phases $A$ and $B$ respectively. The point of intersection of the two lines represents the pressure $P_{\mathrm{TR}}$, at which the free energies of the two phases are equal; $P_{\mathrm{TR}}$ is the thermodynamic equilibrium pressure. The phase $A$ is stable below $P_{\mathrm{TR}}$ whereas phase $B$ is stable above $P_{\mathrm{TR}}$. The symbols $A_{\mathrm{M}}$ and $B_{\mathrm{M}}$ indicate metastable phases.

In the case of a stable equilibrium it is the absolute minimum of the Gibbs free energy. In the case of a local minimum we talk about metastable equilibrium-about metastable phases and states (Chvoj and Kozisek 1991). The lifetime of metastable phases is connected with the height of the energy barrier $\Delta G^{*}$ [see figure 3] which the system must overcome to get from the local extremum. In many cases the lifetime can be as long as thousands of years (e.g. glasses, frozen metastable phases of solid states-diamond, a metastable phase of carbon like fullerene etc). The metastable phases, however, have specific physical properties and are used in technical practice.

Study of kinetics leads to investigation of new metastable phases and Roy (1969) has discussed the impor-

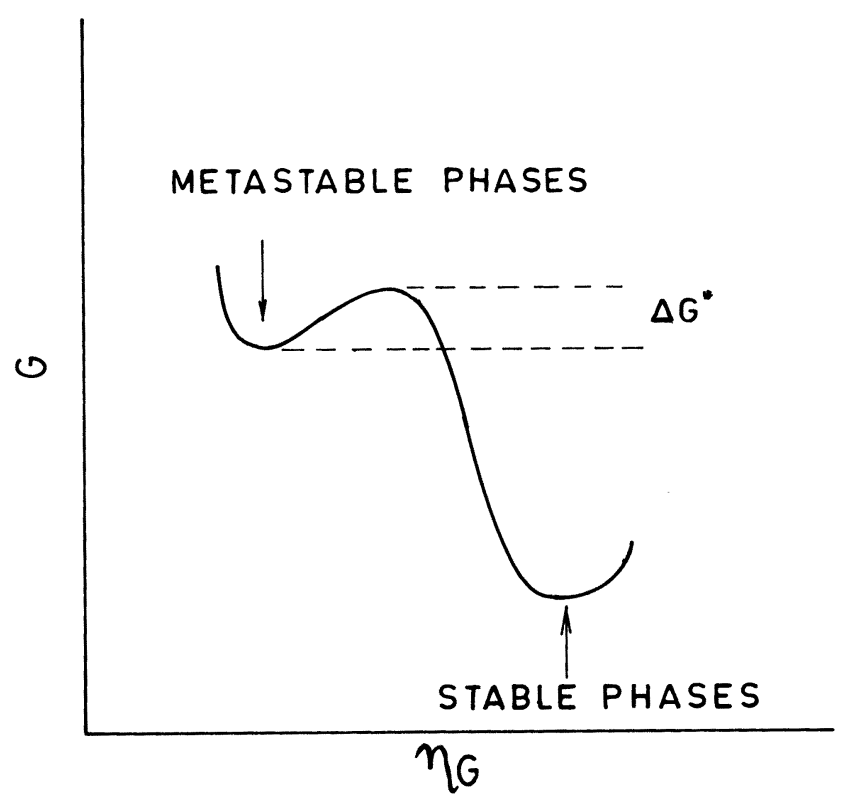

Figure 3. Model dependence of the Gibbs energy $G$ on the order parameter $\eta\left(E_{\mathrm{n}}=\right.$ energy barrier $)$. 
tance of metastable phases in high pressure research. The free energy vs pressure relations between the two polymorphs of a compound is represented by figure $4 \mathrm{a}$. The importance of the activation energy barrier which prevents the metastable phase from reaching the lowest energy state is also crucial and this is expressed in the standard representation of figure $4 \mathrm{~b}$. The metastable phase may undergo finite changes of free energy without transforming into the stable phase. This is true, even when the free energy difference between stable and metastable conditions is very small. Generally the activation energy bears no relation to the free energy differences. In fact, the familiar sketch of figure $4 \mathrm{~b}$ is grossly misleading, since near a transition pressure or temperature, the free energy difference is vanishingly small (at $T_{\mathrm{TR}}, \Delta G=0$ ) whereas the activation energy may be of the order of $10-20 \mathrm{kcal} / \mathrm{mol}$. Two cases with very small and typical activation energies are shown in figure $4 \mathrm{c}$ to represent the situation: the rapid, displacive and the sluggish, reconstructive types of transitions, respectively. In the case of solid phase of the same composition, the most common source of energy whereby a metastable phase transforms to the stable one is usually the thermal excitation energy $k T$. At low temperatures, therefore, it is probable that the same classical entity may form, exist and persist (if not 'forever') for very long times by experimental standards in any one of the several metastable arrangements. It should be noted then, that neither formation, existence, nor persistence is a sufficient criterion as an indicator of thermodynamic stability. In high pressure research, we encounter precisely those conditions which are conducive to the formation of persistent metastable phases. Such routes to metastable phases have attracted much attention because many of the most technologically important and useful materials are metastable. By the use of volume changes, catalysis, shearing stresses and bond breakage, one is able to bring about structural changes under condi- tions where the normal means of attaining the thermodynamically stable arrangement are absent, since $k T$ may be very low. It is, therefore, to be expected that in all high pressure research (especially near room temperature) the probability of metastable reactions occurring and giving rise to metastable phases will be recognized as being very high. Another reason to expect metastable phases in high pressure studies is the high rate with which the intensive variable $(P)$ can be changed in the shock (or dynamic pressure) experiments. This is contrary to the demands of chemical kinetics that during the transport of atoms from one spatial arrangement to another, and time (or the rate) is a most important parameter in real phase transformations. In the case of micro-second pulses to megabar pressures, it is clearly not possible to expect the attainment of thermodynamic equilibrium except in the cases especially favourable. Yet it is not exceptional to find in the pressure literature attempts to find congruence and agreement from static data and those for shock work. Recently high pressure research has picked up momentum in this vital aspect of the problem.

Referring to figure $4 \mathrm{a}$, the transformation $A \rightarrow B$ does not take place at $P_{\mathrm{TR}}$, but often a pressure well above $P_{\mathrm{TR}}$ has to be applied to drive the transformation, due to the presence of an activation barrier. The difference between $P_{\mathrm{TR}}$ and the pressure required to observe $A_{\mathrm{M}} \rightarrow B$ transformation is called over-pressure, and depends on the magnitude of $\Delta G^{*}$ (the activation energy). The larger the $\Delta G^{*}$ the higher will be the over-pressure required for a given rate of transformation. Consequently, any physical property measured with increasing and decreasing pressure around $P_{\mathrm{TR}}$ will exhibit a hysteresis and should be distinguished from the hysteresis effects arising from factors like friction in a high pressure set up. A hypothetical plot of a physical property against pressure exhibiting a hysteresis is shown in figure 5 . These different phases and the magnitude of the property of phase $A$ measured with (a)

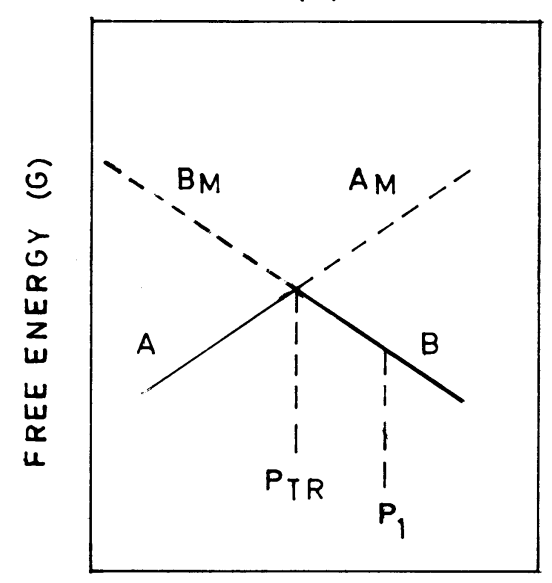

(b)

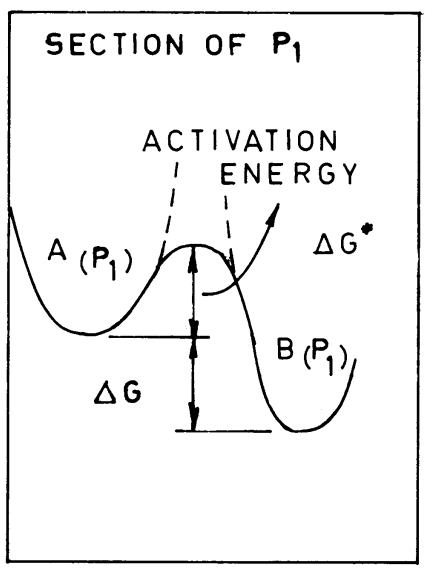

(c)

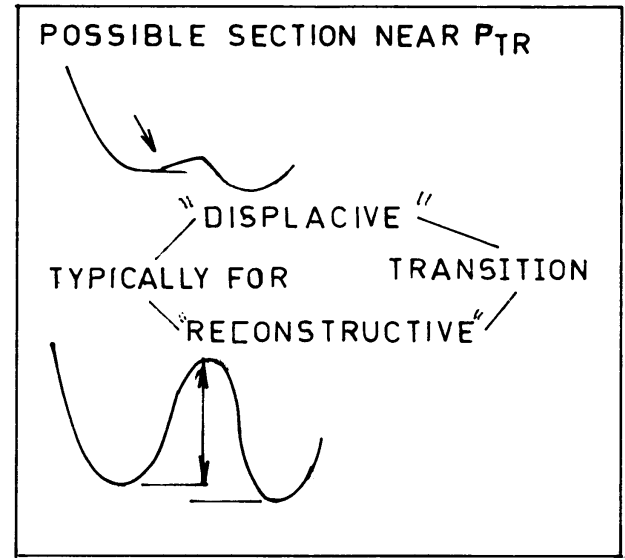

PRESSURE

Figure 4. The familiar representation of the free energy temperature/pressure relations between the two polymorphs of a compound (Roy 1973). 
decreasing pressure does not always coincide with that obtained with increasing pressure. The properties such as electrical conductivity which are sensitive to grain size and the plastic strains in the specimens show on cycling through the transformation, a value which differs from the starting value. However, some other properties such as volume change under pressure show reproducible values with increasing or decreasing pressures in the region where either phase $A$ or $B$ alone is present. It is seen from figure 5 that $P_{\mathrm{TR}}$ lies within the hysteresis loop. This knowledge can be used to experimentally determine $P_{\mathrm{TR}}$ which is an important parameter. When it is assumed that $P_{\mathrm{TR}}$ is located at the midpoint of the hysteresis loop then it is average of the pressures at which $A_{\mathrm{M}} \rightarrow B$ and $B_{\mathrm{M}} \rightarrow A$ are observed to start. As the over-pressure required to produce an observable rate of transformation depends on the activation free energy for the transformation, $P_{\mathrm{TR}}$ can lie at the midpoint of a hysteresis loop only if the activation barriers for the $A_{\mathrm{M}} \rightarrow B$ and $B_{\mathrm{M}} \rightarrow A$ transformations are identical. In general, the position of $P_{\mathrm{TR}}$ remains to be determined by more elaborate experiments.

A method of determining $P_{\mathrm{TR}}$ was suggested by Bridgman (1940). The pressure is increased in small steps and the progress of the transformation is monitored by observing the change in specimen resistivity for a fixed interval of time after each increase. If $A_{\mathrm{M}} \rightarrow B$ transformation is observed, then the pressure is decreased in steps with a waiting period after each decrement of pressure, until transformation is observed. The process is repeated until a region is obtained within which no perceptible $A_{\mathrm{M}} \rightarrow B$ or $B_{\mathrm{M}} \rightarrow A$ transformation takes place. This region was termed as 'region of indifference' by Bridgman. It is then assumed that $P_{\mathrm{TR}}$ lies in the middle of the region of indifference. This method requires that the pressure be

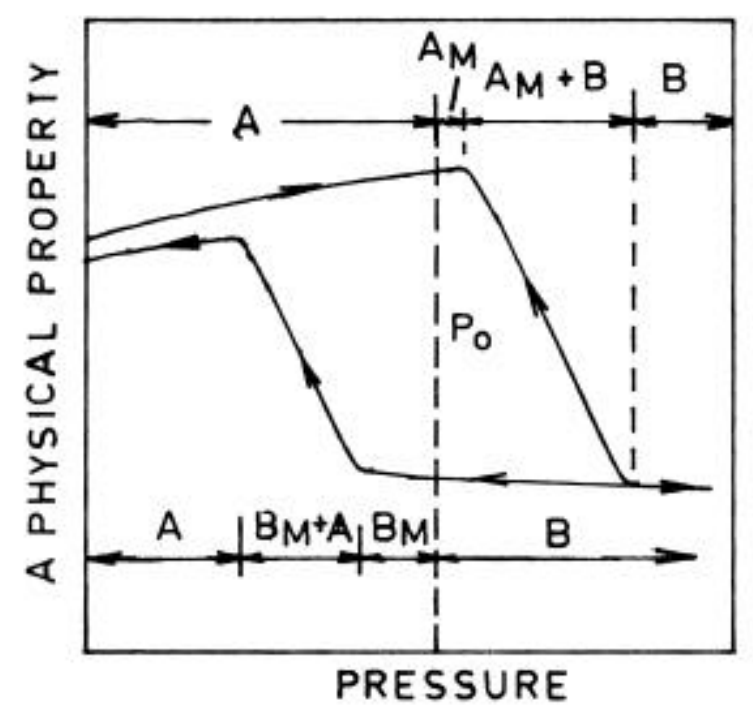

Figure 5. Intrinsic hysteresis when a physical property is measured with increasing and decreasing pressure enclosing $A \rightarrow B$ phase transformation (Singh 1983). truly hydrostatic and be measured in situ by suitable method, for example with the help of a magnanin gauge. This method is time consuming and has been used only for a few transformations which are used as pressure standards. As a result it is customary in high pressure work to quote the pressure at which the transformation is detected with increasing pressure. The transformation pressure thus obtained is termed 'start pressure'. The start pressure is not related to $P_{\mathrm{TR}}$ in any definite manner. The determination of $P_{\mathrm{TR}}$ by the above method breaks down in case of transformations which exhibit such large hysteresis that the high pressure phase is metastably retained at atmospheric pressure. In such cases, the width of the hysteresis can be reduced by intentionally increasing the shear component of strain while pressurizing the specimen.

\section{Theory for the phase change kinetics}

If one looks at the present day scheme of classification by Cohen et al (1979) which identifies broad categories of displacive transformations showing features in common with martensitic transformations but distinct from them. Table 3 is reproduced from a reference (Delaey 1991), showing the classification scheme for the displacive/ diffusionless phase transitions. It is to be noted here that martensitic transformations are here only as a subclass of the broader class of displacive/diffusionless transitions. This review is restricted to only the displacive transformations and the theoretical approach is briefly surveyed.

Displacive/diffusionless reactions are possible only in the solid state and the composition of the product is necessarily the same as that of the original phase. Negligible mixing of the atoms thus takes place during the transformation and it follows that the thermodynamically stable configuration of the assembly often cannot be produced by such a change. Although the transformation are called displacive, the formation of stable small regions of product and the subsequent growth of these nuclei may have to be treated as separate stages in both classes of transformation.

One of the classification schemes (see table 4) (Rao and Rao 1978) focuses on the growth process but either the growth rate or the nucleation rate may be effective in determining the overall kinetics of a heterogeneous transformation. The net transformation rate will depend mainly on the slower of the two stages becoming virtually zero if either the nucleation rate or the growth rate is sufficiently slow. We should thus consider whether or not there are differences in the physical mechanisms leading to the formation of nuclei in particular transformation. If there are such differences, a classification of transformation based on the nucleation stage will be equally valid. There are various ways in which nuclei may be formed, but these are not readily distinguished by experimental criteria. The growth classification is convenient largely because most 
reactions in the solid state are greatly influenced by the growth mechanism.

This formal theory is largely independent of the particular models used in the detailed descriptions of the mechanisms of transformation and therefore is given here. Consider a first order phase transition $\alpha \rightarrow \beta$. This transition, in general, does not occur homogeneously; rather small domains of the phase $\beta$ first become distinct at various points in $\alpha$ separated by many molecular spacings. These domains then propagate at the expense of $\alpha$. This mode of reaction is known as nucleation and growth, the birth of domains being called nucleation.
Thus, the total rate of a phase change $\mathrm{d} x / \mathrm{d} t$, where $x$ is the fraction of $\alpha$ transformed isothermally to $\beta$ in the time $t$, is determined by two constants: the rate of nucleation $I$ of $\beta$ domains and their rate of growth $u$ after nucleation. Both these constants must be known in order to establish a unique interpretation of data on the rate of phase change.

The rates of nucleation and growth can be combined, with due correction for the impingement of $\beta$ domains, to give the isothermal kinetic law $x=f(t)$ governing phase transformation. Some of the simpler derivations are given here.

Table 3. Cohen's classification table (Cohen et al 1979).

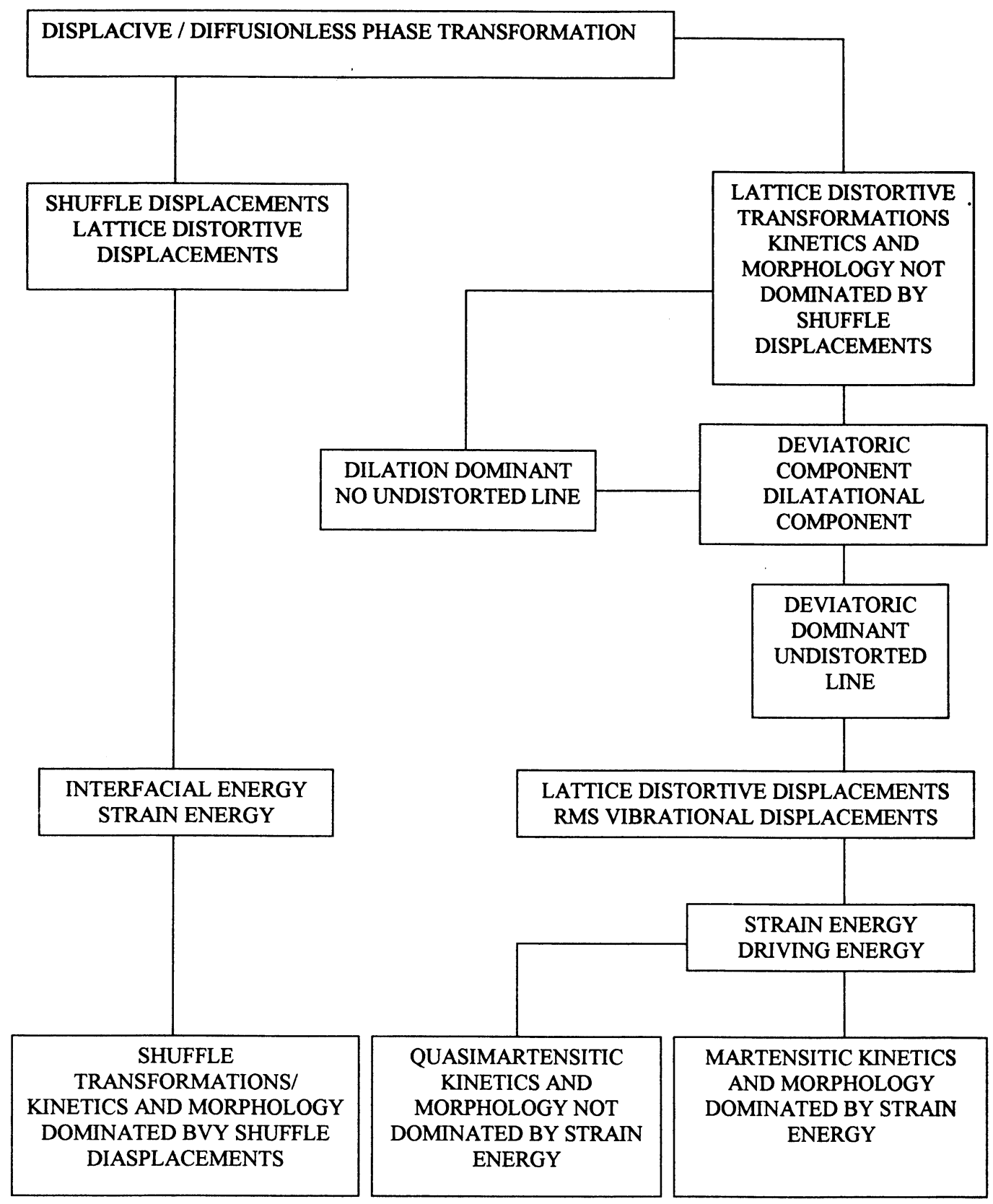


Johnson and Mehl (1939), Avrami (1939, 1940, 1941), Burke and Turnbull (1952) and Turnbull (1956) have given the derivations of the kinetic law for transformation in which the $\beta$ growth rate is time independent, as will generally be the case unless growth is controlled by diffusion of a component through $\alpha$. The volume $v$ of a $\beta$ domain growing at a constant linear rate and before impingement or growth barriers, such as other domains or free surfaces, is given by

$$
v=g u^{3}(t-\tau)^{3}
$$

where, $g$ is a shape factor, $t$ the total transformation rate and $\tau$ the time since the beginning of transformation at which the domain is nucleated.

An extended volume $x_{\mathrm{ex}}$ is defined to be the total volume of all $\beta$ domains calculated without regard to impingement and including the volume contributed by the 'phantom' domains which would nucleate in regions already transformed. Thus,

$$
x_{\mathrm{ex}}=\int_{0}^{t} v I d=g u^{3} \int_{0}^{t}(t-\tau)^{3} I \mathrm{~d} \tau,
$$

$x_{\text {ex }}$ is simply related to the total transformed volume as follows

$$
x=1-\exp \left[-x_{\mathrm{ex}}\right]
$$

where the value of the system is assumed to be unity for all $x$ values. This relationship was first arrived at intuitively by Johnson and Mehl (1939) and later justified rigorously by Avrami (1940, 1941). Combination of (2) and (3) gives the basic kinetic law

$$
x=1-\exp \left[-\int_{0}^{t} v I \mathrm{~d} \tau\right]
$$

Johnson and Mehl (1939) and Avrami (1940, 1941) have carried out the integration indicated in (4), after making various assumptions about the dependence of the nucleation frequency $I$ on time. For example, if $I$ is time independent then (4) becomes

$$
x=1-\exp \left[-g u^{3} I t^{4} / 4\right]
$$

More generally, $I$ is time dependent and, in the event, the kinetic data can often be described approximately by the equation

$$
x=1-\exp \left[-b t^{n}\right]
$$

where $3 \leq n \leq 4$ for $I$ decreasing with $t$, and $n>4$ for $I$ increasing with $t$.

Table 4. Relations between various transformations in metal systems (Rao and Rao 1978).

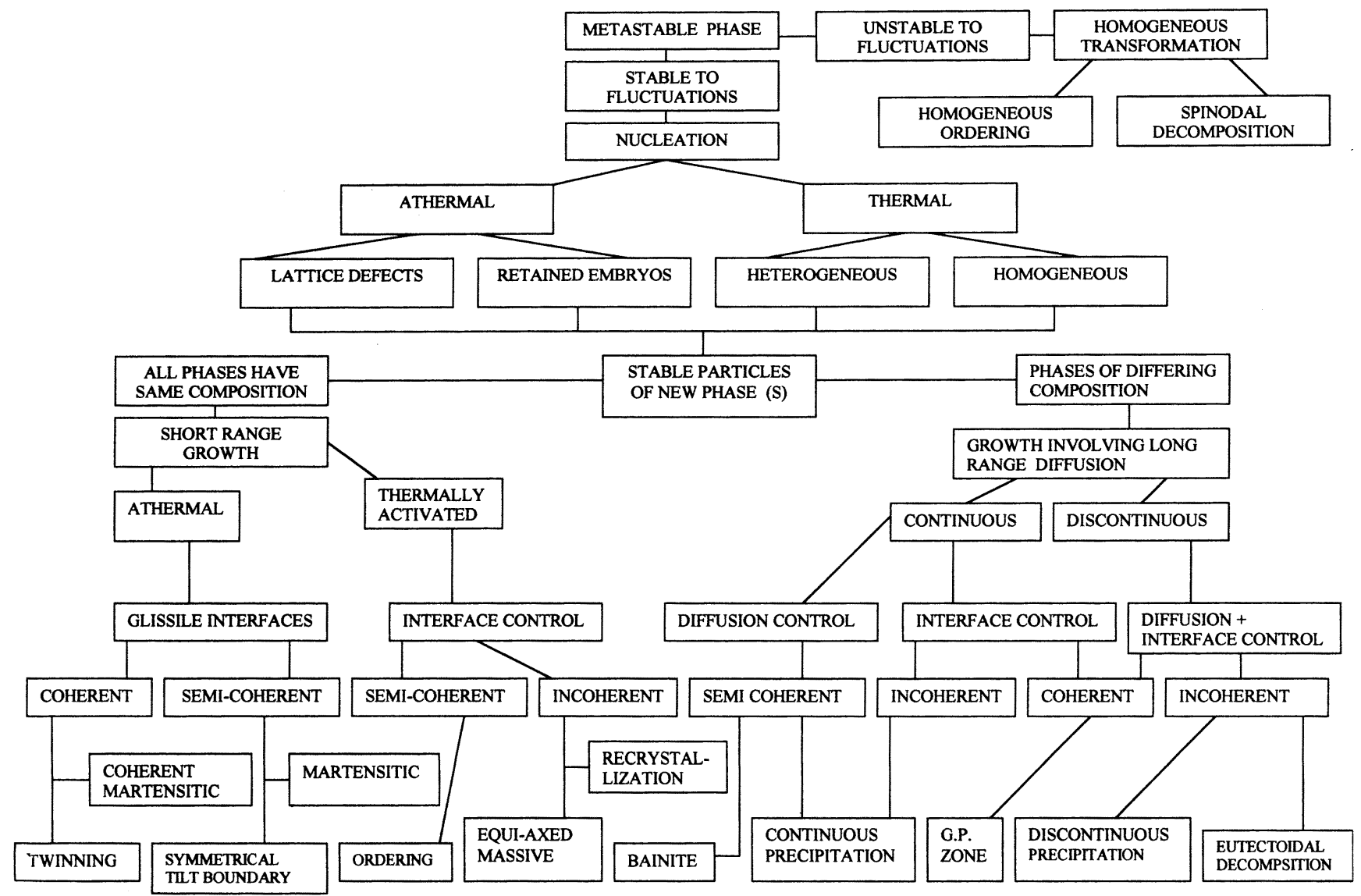


Various other factors may modify the form of the kinetic law for constant growth rate. For example, if, after nucleation is complete, the internuclear spacing $\delta$ equals or exceeds one or two dimensions of the $\alpha$ specimen, $n$ in (6) is diminished by one or two units, respectively. Also, the $\alpha$ structure is often divided into domains or grains by internal surfaces that are preferred sites for the nucleation of $\beta$. If the number $N_{\beta}$ of $\beta$ nuclei that form is no greater than the original number $n_{\alpha}$ of domains, the kinetic law is well represented by (4). Johnson and Mehl (1939) and Cahn (1956) have developed the kinetic description of the more complicated cases where $N_{\beta}>n_{\alpha}$.

The isothermal transformation curves obtained by substituting $n=1$ in (6) is equivalent to that for a first order homogeneous reaction. All the other possibilities however, give sigmoidal curves for $x$ against $t$, in which the fractional volume transformed increases slowly at first, then much more rapidly, and, finally slowly again. Most experimental transformation curves are sigmoidal in shape; a typical example is shown in figure 6. Avrami (1940, 1941) pointed out that if we plot $x$ against $\ln (t)$, all the curves with the same value of $n$ will have the same shape, and will differ only in the value of $b$, which is equivalent to a change of scale. He therefore proposed that the 'shape' of a reaction curve should be defined by a $x-\ln (t)$ plot. A more useful plot is $\log \log [1 /(1-x)]$ against $\log (t)$ the slope of which gives $n$.

In (6), the above theory applies to many real transformations. However, it cannot be safely applied in practice unless the assumptions that the rate of nucleation is constant and growth is isotropic can be verified. The Avrami theory assumes that the nucleation frequency is either constant, or else is maximum at the beginning of transformation and decreases (slowly or rapidly) during the course of transformation. In contrast to this, there may be an operational rate of nucleation which increases with time. The Avrami equation is also often applied to transformations in which the growth is diffusion controlled, but in most cases there is no adequate theoretical sanction for this.

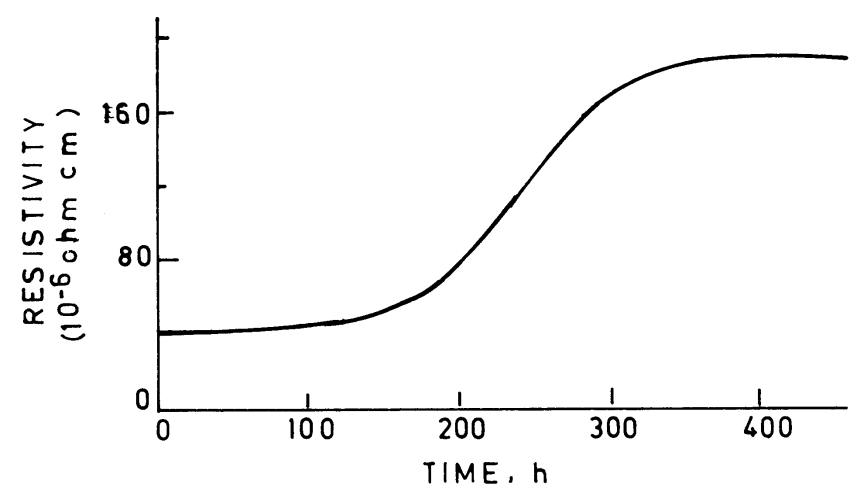

Figure 6. Kinetics of transformation $\beta \rightarrow \alpha$ at $25^{\circ} \mathrm{C}$ as shown by electrical resistivity measurement (Christian 1975).
Table 5 summarizes the values of $n$ which are obtained in various experimental situations. The conditions listed are not meant to be exhaustive, for example the effects of an external surface which may reduce $n$ by up to 1 for a foil and 2 for a wire have not been included. It is evident from table 5 that a kinetic investigation that is limited to the establishment of the assumed growth law does not, as once assumed, give sufficient information for the growth habit to be deduced.

\subsection{Theory of heterogeneous nucleation}

The kinetics of a heterogeneous reaction can usually be described in terms of the separate nucleation and growth of the transformed regions. The classical theory of nucleation by random fluctuation in a metastable assembly is due mainly to Volmer (1926) and Becker and Doring (1935), but many other workers have made significant contributions. This theory was formulated first for the simplest nucleation process, the condensation of a pure vapour to form a liquid, and this served as a stepping stone for more complex problems of nucleation in liquid and solid phases. Detailed theory is dealt in the excellent text by Christian (1975).

It is well known that certain structural singularities, such as impurity particle and grain boundaries, may strongly promote (or catalyse) the nucleation of phase change (Turnbull 1950; Hollmon 1950). The basis for the formal description of this 'catalysis of nucleation' was laid by Gibbs.

Consider the formation from $\alpha$ of a small $\beta$ domain on the surface of an impurity particle suspended in $\alpha$. Whether or not the impurity surface catalyses $\beta$ nucleation is determined by the magnitude of $W_{\mathrm{S}}$, which is the net free energy of formation in unit area of interface between $\beta$ and impurity. That is

$$
W_{S}=\sigma_{\beta S}-\sigma_{\alpha S},
$$

where $\sigma_{\alpha S}$ and $\sigma_{\beta S}$ are the $\alpha$-impurity and $\beta$-impurity interfacial tensions, respectively. The impurity surface will be a preferred (relative to the body of $\alpha$ ) $\beta$ nucleation site, provided $W_{\mathrm{S}}$ is less than the interfacial tension $\sigma_{\alpha \beta}$ between $\alpha$ and $\beta$. The greater are the intermolecular

Table 5. Values of $n$ in kinetic law in polymorphic changes (Christian 1975).

\begin{tabular}{lr}
\hline Conditions & $n$ \\
\hline 1. Increasing nucleation rate & $>4$ \\
2. Constant nucleation rate & 4 \\
3. Decreasing nucleation rate & $3-4$ \\
4. Zero nucleation rate (saturation of point sites) & 3 \\
5. Grain edge nucleation after saturation & 2 \\
6. Grain boundary nucleation after saturation & 1 \\
\hline
\end{tabular}


attractive forces between $\beta$ and impurity, the less is $W_{\mathrm{S}}$. If the impurity interacts more strongly with $\beta$ than with $\alpha$, $W_{\mathrm{S}}$ is negative.

Assuming the applicability of the Gibbs theory of surfaces, the free energy of formation $\Delta G_{\mathrm{S}}$ of a $\beta$ cluster containing $i$ molecules from $\alpha$ on an impurity surface is, in general, given by,

$$
\Delta G_{\mathrm{S}}=i^{2 / 3} \Sigma\left[a_{\beta \mathrm{S}} W_{\mathrm{S}}+a_{\alpha \beta} \sigma_{\alpha \beta}\right]+v i\left(\Delta G_{\mathrm{V}}+E\right),
$$

where $a_{\beta s} i^{2 / 3}$ and $a_{\alpha \beta} i^{2 / 3}$ are areas of facets in the $\beta$ impurity and $\alpha-\beta$ interfaces, respectively. The summation is over all facets of the cluster. For a given impurity surface, $\Delta G_{\mathrm{S}}$ is dependent upon the shape and the state of strain of the cluster as well as $i$. The characteristics of the impurity particle which affect $\Delta G_{\mathrm{S}}$ are $W_{\mathrm{S}}$, which is a function of the crystallographic orientation of the impurity surface, and its radius of curvature $r_{\mathrm{S}}$, if $\left|r_{\mathrm{S}}\right| \leq r$ (where $r$ is the cluster radius).

When $\left|r_{\mathrm{S}}\right| \gg r$ and $\sigma_{\alpha \beta}>\left|W_{\mathrm{S}}\right|, \Delta G_{\mathrm{S}}$ for any given cluster shape, goes through a maximum $\Delta G_{\mathrm{S}}^{*}$ with increasing $i$. In order to illustrate the calculation of the nucleus shape for which $\Delta G_{\mathrm{S}}^{*}$ is a minimum, we shall assume, for simplicity, that $\sigma_{\alpha \beta}$ and $W_{\mathrm{S}}$ are independent of boundary orientation and the nucleus strain is a constant. Equation (8) then reduces to

$$
\Delta G_{\mathrm{S}}=A_{\beta \alpha} W_{\mathrm{S}}+A_{\alpha \beta} \sigma_{\alpha \beta}+v i\left(\Delta G_{\mathrm{S}}+E\right),
$$

where $A_{\beta \alpha}$ and $A_{\alpha \beta}$ are the areas of the cluster in contact with the impurity surface and $\alpha$, respectively. For a given $i, \Delta G_{\mathrm{S}}$ is a minimum when the cluster has the shape of a spherical cap making a contact angle $\theta$ (see figure 7) with the impurity surface. This contact angle is related to $W_{\mathrm{S}}$ by the equation

$$
\cos \theta=-W_{\mathrm{S}} / \sigma_{\alpha \beta}
$$

It follows that
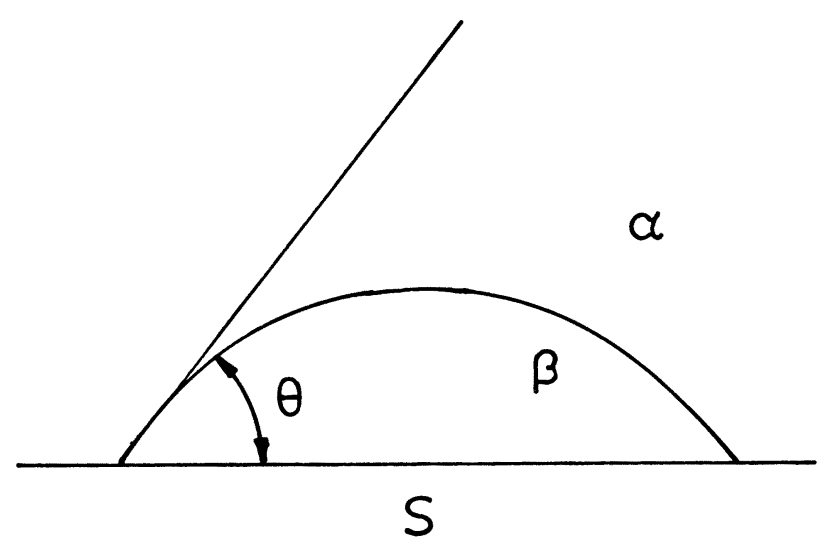

Figure 7. Element of phase $\beta$ (from $\alpha$ ) with a contact angle $\theta$ on the surface of an impurity particle $S$ (Turnbull 1956).

$$
\Delta G_{\mathrm{S}}^{*}=16 \pi\left(\sigma_{\alpha \beta}\right)^{3} f(\theta) / 3\left(\Delta G_{\mathrm{V}}+E\right)^{2}=\Delta G^{*} f(\theta),
$$

where $f(\theta)=(2+\cos \theta)(1+\cos \theta)^{2} / 4$ and $\Delta G^{*}$ the thermodynamic barrier to nucleation (at constant strain) in the body of $\alpha$. Depending on $W_{S}$, the magnitude of $f(\theta)$ ranges between unity and zero. The limiting values are $f(\theta)=1$ when $W_{\mathrm{S}}=\sigma_{\alpha \beta}$ and $f(\theta)=0$ when $W_{\mathrm{S}} \geq \sigma_{\alpha \beta}$.

The frequency of nucleation $I_{\mathrm{S}}$ can be derived as (Turnbull 1952)

$$
I_{\mathrm{S}}=K_{\mathrm{S}} \exp \left[-\left(\Delta G_{\mathrm{S}}^{*}+\Delta G_{\mathrm{a}}^{\prime}\right) / k T\right]
$$

where

$$
K_{\mathrm{S}}=n^{*}\left(\sigma_{\alpha \beta} / k T\right)^{1 / 2}(2 v / 9 \pi)^{1 / 3}\left[f(\theta)^{1 / 6}\right] n_{\mathrm{s}} v_{\mathrm{o}},
$$

$n^{*}$ is the number of molecules in the nucleus in contact with $\alpha$ and $n_{\mathrm{S}}$ the number of molecules per unit area from $\alpha$ in contact with the impurity surface.

It follows from the theory of solid interface boundaries that the effectiveness of an impurity in nucleating $\beta$ should depend markedly on the relation between the crystal structures of impurity, $\alpha$ and $\beta$. Also the reasons why grain boundaries may be preferred as nucleation sites, may be (i) diffusive processes in nucleation are much more rapid at grain boundaries than within the crystal, (ii) relief of transformation stresses in nuclei by flow can occur more rapidly at grain boundaries than elsewhere and (iii) impurity particles which might catalyse nucleation, are concentrated at the grain boundaries. Dislocations also should be favoured sites for the nucleation of certain phase changes. According to the theory, the nucleation frequency in crystals should be strongly dependent upon $\alpha$ structure and hence on the state of strain of $\alpha$.

\section{Experimental review}

Inspite of the voluminous literature on the phase equilibria studies under pressure few investigations have been reported on the kinetics of phase transitions under pressure. Bridgman (1916) carried out first such a study, in which various solid-solid transition rates were studied by measuring the rate of fall or rise of pressure toward the equilibrium lines due to the volume change accompanied by the transitions. In his work the rate increased rapidly as the pressure was displaced from the pressure of equilibrium between the phases. The ideal would be to obtain in situ X-ray diffraction pattern while the transition proceeds under pressure. Various other methods have been applied to the study of kinetics of high-pressure phase transformations. Selection of the appropriate method depends on the properties of the sample under consideration. In the following, some of the well known methods are briefly reviewed. For earlier reviews on the kinetic aspects of phase transition, see Onodera (1972), Singh (1983) and Lorenz (1992). 


\subsection{Scattering experiments}

Structural phase transitions are investigated by means of $\mathrm{X}$-ray or neutron scattering. The high and low pressure structures are well distinguished by their characteristic diffraction patterns. Comparing the peak intensities of both the phases, the transformation degree $x(t)$ (the amount of high pressure phase) as a function of time can be obtained. This method has been successfully applied to study of kinetics of pressure induced phase transitions in $\mathrm{PbO}_{2}$ (Dachlli and Roy 1961), $\mathrm{KCl}$ (Hamaya and Akimoto 1981), $\mathrm{GeO}_{2}$ (Zeto and Roy 1969; Brar and Scloessin 1981), $\mathrm{Fe}_{2} \mathrm{O}_{3}$ (Goto 1964), RbI (Hamaya et al 1986), La (Merkau and Holzapfel 1986; Kruger et al 1990) and other compounds.

The main shortcoming of the scattering method for kinetics investigations consists in the limited time resolution due to the comparatively long period needed for measurement. The time resolution is improved by using the high intensity of synchrotron radiation. However, the method is not applicable to fast transitions. In order to calculate the amounts of high and low pressure phases, the measured intensity have to be normalized with respect to the spectra of the pure phases. This reduced the accuracy in determining quantity $x(t)$ which is commonly fitted to the Avrami equation for nucleation and growth model. The information obtained from the transformation degree $x(t)$ is not sufficient to understand the nucleation and growth kinetics. The parameters $b$ and $n$ in the Avrami equation have unique physical meaning only in some special cases.

\subsection{Conductivity measurements}

Electrical conductivity/resistivity is a valuable tool for studying phase transformations in metals and semiconductors under pressure (Rajan et al 1986). In the transition region the measured conductivity is an averaged quantity $\sigma_{\alpha \mathrm{V}}$ depending on the percentage of high and low pressure phases and on the distribution of both phases in the sample. In order to calculate the transformation degree $x(t)$ from $\sigma_{\alpha \mathrm{V}}$ an effective medium model has to be applied. For a random mixture of the two phases with conductivities $\sigma_{1}$ and $\sigma_{2}$ and percentages $x_{1}$ and $x_{2}$ respectively the averaged conductivity is determined by the equation (Landauer 1952)

$$
\left[x_{1}\left(\sigma_{1}-\sigma_{\alpha \mathrm{V}}\right) /\left(\sigma_{1}+2 \sigma_{\alpha \mathrm{V}}\right)\right]+\left[x_{2}\left(\sigma_{2}-\sigma_{\alpha \mathrm{V}}\right) /\left(\sigma_{2}+2 \sigma_{\alpha \mathrm{V}}\right)\right]=0
$$

This formula has been used to investigate the kinetics of high pressure phase transitions by means of conductivity measurements (Singh et al 1983b; Singh 1985).

The method described above is not limited in its time resolution. Therefore it is suitable for the observation of fast transition kinetics. The averaged conductivity $\sigma_{\alpha \mathrm{V}}(t)$, however, depends upon the distribution of the high and low pressure phases in the sample and the appropriate model has to be used to calculate $x(t)$ from $\sigma_{\alpha \mathrm{V}}(t)$. Thus the calculated $x(t)$ may be model dependent.

\subsection{Quenching of high pressure phases}

High pressure phases in solid frequently remain metastable after the release of pressure. This opens the possibility of quenching samples in different kinetic stages of a phase transformation. Various physical and chemical methods can be applied to investigate the quenched systems under ambient conditions. Besides the transformation degree $x$ in the given stage on the transition further characterizes quantities like the grain structure (size, shape and distribution) of the high and low pressure phases. This information yields more insight into the basic mechanisms driving the phase transitions.

The quenching method has been successfully applied, for example to study the olivine-spinel transition (Sung 1979) and to explain the basic mechanism of the high pressure synthesis of cubic boron nitride (Lorenz and Lorenz 1989). The shortcomings of the method consists in the large number of experiments with different samples which are required to investigate the transition kinetics with sufficient accuracy. Since the transition rate $x(t)$, according to the Avrami equation, varies strongly with time it is more convenient to perform experiments with a fixed duration and to consider the pressure and temperature dependence of the kinetic parameters (nucleation and growth rates). If the sample undergoes some substantial changes during quenching the results should be considered more carefully.

\subsection{Kinetics of phase transition in a diamond anvil cell $(D A C)$}

Research into the properties of solids by means of DAC has greatly increased during the past few years. Due to its small size, the DAC can be modified to suit most experimental requirements. These various adaptations have been subject of many review articles (Jayaraman 1983, 1986; Yousuf 1998).

Kinetics of structural phase transitions have been investigated for various solids in a DAC (Brar and Scloessin 1981; Merkau and Holzapfel 1986; Kruger et al 1990) as well as in other high pressure devices (Hamaya and Akimoto 1981; Hamaya et al 1986). The essential problems of the diffraction methods lie in the interpretation of the measured transformation degree $x(t)$ and its fit to the Avrami equation without knowing further characteristics quantities like nucleation rates or growth velocities. Avrami equation is valid for fixed thermodynamic conditions (pressure and temperature) and the value of the 
exponent $n(1 \leq n \leq 4)$ depends on the growth kinetics. In the pressure chamber of the DAC, however, it is well known that the pressure changes during a phase transition due to the volume change of the sample (Hirsh and Holzapfel 1983) and other effects related to the sample preparation technique (Dassler 1990). Due to this pressure change the validity of the Avrami equation and the physical meanings of the parameters $k$ and $n$ are questionable.

The DAC apparatus for the high pressure generation gives the possibility of qualitative as well as quantitative optical investigations of the kinetics of phase transformations in solids under pressure. The diamond anvils are transparent for optical radiation over the range 0.2-5.0 eV (Welber 1976). This makes it possible for us to consider a rich variety of spectroscopic experiments as well as visual and video observations. The knowledge of such transformation behaviour is of great importance to understand its mechanism. The interpretation of the transformation process requires determination of different kinetic parameters with a high time resolution, as well as an accurate pressure determination.

Dassler (1988), Dassler and Riedel (1992), and Riedel and Dassler (1990a) have described an apparatus and a new optical method for quantitative study of various parameters of a phase transition. The determination of the degree of transformation with a maximum time resolution of $1 \mathrm{sec}$ is made possible by a direct image processing procedure. Other kinetic parameters, such as the nuclea- tion rate and the grain size distribution, are measured with a maximum time resolution of $30 \mathrm{sec}$ after an image data transfer in conjunction with external image processing and data storage. The investigation of the transformation process in the compressed sample between the diamond anvils is achieved by video camera observation followed by a digital image processing procedure. The pressure in DAC is determined in situ from the shift of the R-line fluorescence of a ruby crystal. Riedel and Dassler (1990b) also report the investigation of phase transition in $\mathrm{KCl}$.

The apparatus developed by Dassler (1988) consists of four main components: the high pressure DAC for pressure generation; a fluorescence microscope modified for a laser input and three directional signal output, an image processing system for image monitoring and image colouring, combined with a digitizer for image transfer to an external computer; and an optical multichannel analyser (OMA) for spectral measurements of luminescence and absorption set up illustrated in figure 8 .

The high pressure phase transformation in alkali halides from the $\mathrm{NaCl}(B 1) \rightarrow \mathrm{CsCl}(B 2)$ has been experimentally studied by a variety of methods, but only a few measurements have been made concerning the kinetics (Hamaya and Akimoto 1981). The results of DAC (image processing) and X-ray measurements are compared in figures 9 and 10 (Dassler 1988). It can be seen that transition behaviour is qualitatively similar. The energy dispersive $\mathrm{X}$-ray diffraction (EDXD) studies however, show comple-

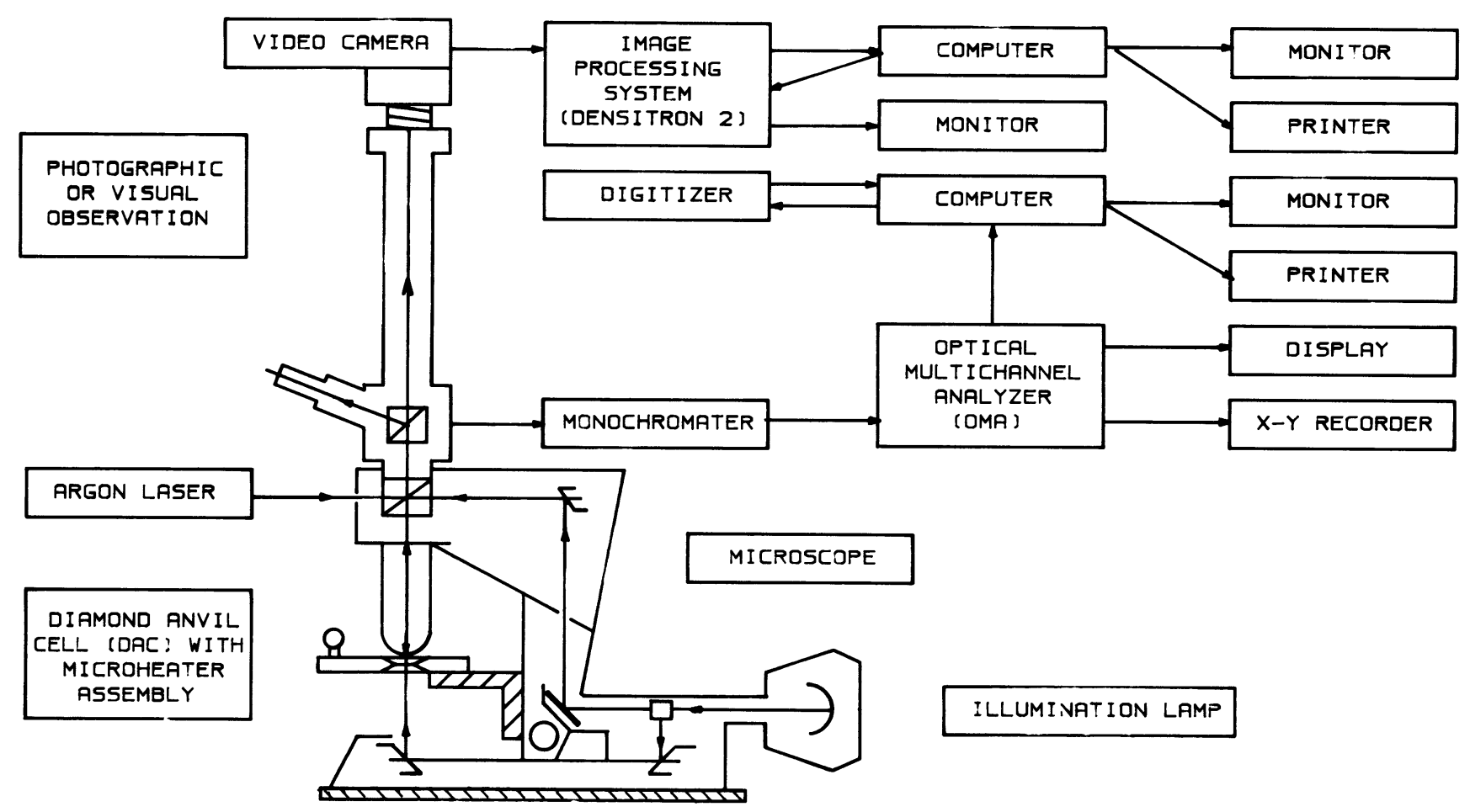

Figure 8. Schematic diagram of the experimental set up for studying phase transformation in the diamond anvil cell (Dassler 1988). 
tion of transformation in a shorter time. The lower transformation velocity in the DAC experiments, compared with X-ray investigations, underlines the strong dependence of the kinetics measurements on the preparation procedure. Therefore the relative importance of the various nucleation and growth parameters will clearly vary during the transition. Therefore the whole transformation process has to be investigated in more detail. The precipitation of the solid phase is governed by a nucleation and growth process with changing pressure. Thereby, after raising the force of the press, an initial pressure increases due to the plastic deformation of the gasket material followed by a decrease depending on the transition to the denser phase. Because of the strong pressure change during the transition an interpretation of the experimental data is ruled out.

Lorenz and Orgzall (1991) have presented a new method which is suitable to interpret the phase transition kinetics under changing pressure conditions and even more complicated situations. The procedure makes use of the experimentally determined nucleation rate $I(t)$ and the transformation degree $x(t)$ as an input for a numerical simulation of the transition. They have derived the following expression for time dependent growth process in a DAC

$$
\ln [1-x(\tau)]=-\alpha v_{0}^{2} \beta \int_{0}^{t} I(\tau)(\tau-\tau)^{\mathrm{m}} \mathrm{d} \tau,
$$

where the problem consists of calculation of $I(\tau)$ from the measured functions $I(t)$ and $x(t)$.

With the time scaling nucleation and growth problem under the changing pressure conditions in a DAC is mapped to the much simpler problem of nucleation and growth, where external conditions are fixed and the effective nucleation rate is given by $I(\tau)$.

6.4a Behaviour of RDX: The thermophysical and thermochemical properties of RDX, 1,3,5-trinitro hexa-

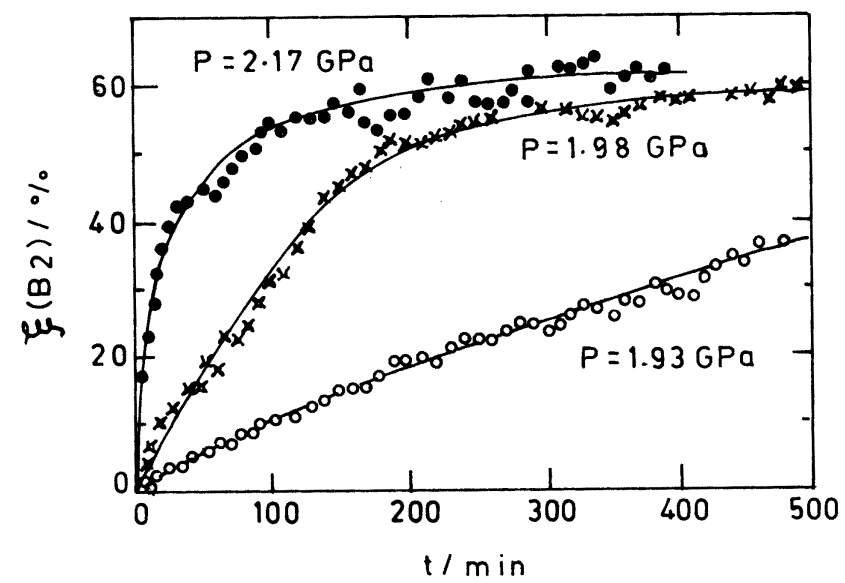

Figure 9. Plots of the degree of transformation, given as the percentage of the $B 2$ phase present in $\mathrm{KCl}$, determined by image processing, against time for three different values of excess pressure (Dassler 1988). hydro-1,3,5 triazine, at elevated pressures are of interest because this material is used extensively in monopropellants and explosives. Understanding of the pressure effect is necessary for accurate modelling of combustion and explosive behaviour. Miller et al (1991) studied the effects of pressure on the thermal decomposition kinetics, chemical reactivity and phase behaviour of RDX by a combination of measurement techniques in conjunction with a high pressure diamond anvil cell. These techniques include (i) Fourier transform infrared (FTIR) spectroscopy for kinetic measurements and phase identification and (ii) energy dispersive X-ray powder diffraction (EDXD). EDXD is used for identification of the observed polymorphic transitions. Optical polarizing microscopy is employed for visual detection, confirmation of phase transformations and determination of transition pressures. Studies were limited to the region where decomposition rates could be measured within reasonable laboratory time i.e. below $10 \mathrm{GPa}$ and $573 \mathrm{~K}$. The $P-T$ phase diagram for RDX was determined to be $573 \mathrm{~K}$ and $7 \cdot 0 \mathrm{GPa}$, delineating the stability fields of three solid phases $\alpha, \beta$ and $\gamma$ and the liquids. The $\alpha$ and $\beta$ phases of RDX were found to thermally decompose, while the $\gamma$ phase transformed to either $\alpha$ or $\beta$ before reaching decomposition temperature. The decomposition rate of $\alpha$ phase was found to increase with increasing pressure suggesting a bimolecular-type mechanism. An activation energy of $51 \mathrm{kcal} / \mathrm{mol}$ and activation volume of $-5.6 \mathrm{~cm}^{3} / \mathrm{mol}$ were calculated for the thermal decomposition reaction of $\alpha$ RDX.

6.4b Oil generation kinetics and kerogen: The diamond anvil cell has been used to study hydrothermal reactions (Bassett et al 1996). Huang (1996) adopted this technique in visualizing kerogen transformation during pyrolysis. Also they have (Huang and Otter 1998) determined the oil generation kinetics by DAC-fluorescence and infrared spectroscopy (FS/IR) pyrolysis. The results

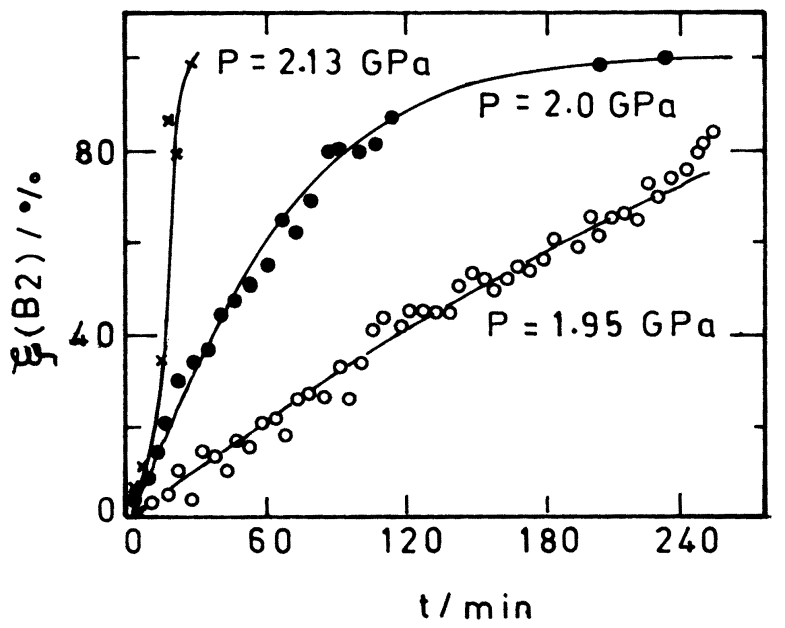

Figure 10. Typical plots of the degree of transformation given as the growth of the $B 2$ phase, against time, at three different constant pressures (Dassler 1988). 
suggest that this technique may be used to determine the kinetic parameters for oil generation from source rocks. The absorption of early generated liquid in the samples plus early generation of nonfluorescing petroleum, most likely gases, is a possible explanation. The results of the DAC-IR experiments seems to have confirmed the capabilities of the in situ technique for examining the reaction pathways, the timing and the rate of the organic transformation.

\section{Past experience on kinetics of phase transitions}

The published experience on transformation kinetics is vast. But if one looks from a narrow window of 'pressure induced structural transformations' then the count dwindles down. It was very difficult to find adequate material to give a variety of examples. Only those experiments are reviewed which are most pertinent to high pressure field. Carbon is chosen because of the success of diamond synthesis and the way it has been generating the researchers interests till today. $\mathrm{GeO}_{2}$ is given as an example of system studied using scattering experiments/pressure quenching, $\mathrm{Ti}, \mathrm{Yb}$ and $\mathrm{Bi}$ for conductivity studies. Amorphization under pressure is reviewed briefly because of recent resurgence and promises it shows for studies on kinetics.

\subsection{Carbon}

Diamond synthesis is the oldest and the best example of a success story which depended heavily on reaction kinetics. Although the element carbon has relatively simple and symmetrical atoms with four valence electrons, the forms it can take in the solid, liquid and gaseous states can be of considerably complicated variety. There are innumerable kinds of carbon viz. blacks, graphites, fullerenes as well as many types of diamonds. Very little is known about the structure and nature of liquid carbon (Bundy 1969).

The graphitization of diamond was first reported by Bridgman (1947), in which diamond crystals were embedded in graphite within a high pressure apparatus and transiently heated by a thermite mixture while held under various ambient high pressures ranging from 1.5 to $3 \mathrm{GPa}$. The reaction kinetics obtained from these experiments provided rough estimation of the $P, T$ location of the equilibrium line.

Results of extensive high pressure-high temperature experimental studies of graphitization of diamond over a range of pressures from vacuum up to over $3 \mathrm{GPa}$ at temperature up to $2500 \mathrm{~K}$ were used (Bundy 1969; Gomon et al 1974) to plot log (heating time) versus $10 / T \mathrm{~K}$, with data circles blacked out by a functional amount corresponding to the reactions of graphitization (figure 11). From the slope of such lines an activation energy could be derived from the relationship

$$
\ln \tau=Q^{*} / R(I / T)+\text { const., }
$$

which is derived from the simplified rate relationship

$$
\text { Rate }=C \exp \left(-Q^{*} / R T\right)
$$

where $\tau$ is the time at temperature $T, Q^{*}$ the activation energy, $R$ the molar heat constant and $C$ is a constant which includes collision frequency and nucleation terms.

The data indicated activation energies of 175 and $256 \mathrm{kcal} / \mathrm{mole}$ for $0-2 \mathrm{GPa}$ pressures, respectively. The vacuum value of $175 \mathrm{kcal} / \mathrm{mole}$ coincides with a widely accepted value for the vaporization energy of graphite (or of diamond also, because the free energy difference between diamond and graphite is relatively small). Results indicated that the activated state between diamond and graphite was essentially vapour. If this were the case such a mechanism under high pressure would require considerable PV work against the environment to obtain the activated state. Even if the pressure increases, activation energy too would increase causing the reaction rate to become negligibly small.

A plausible alternative was to find some kind of solvent catalysis material which would reduce the activation energy considerably and make the reaction less sensitive to the ambient pressure. Efforts in this direction were

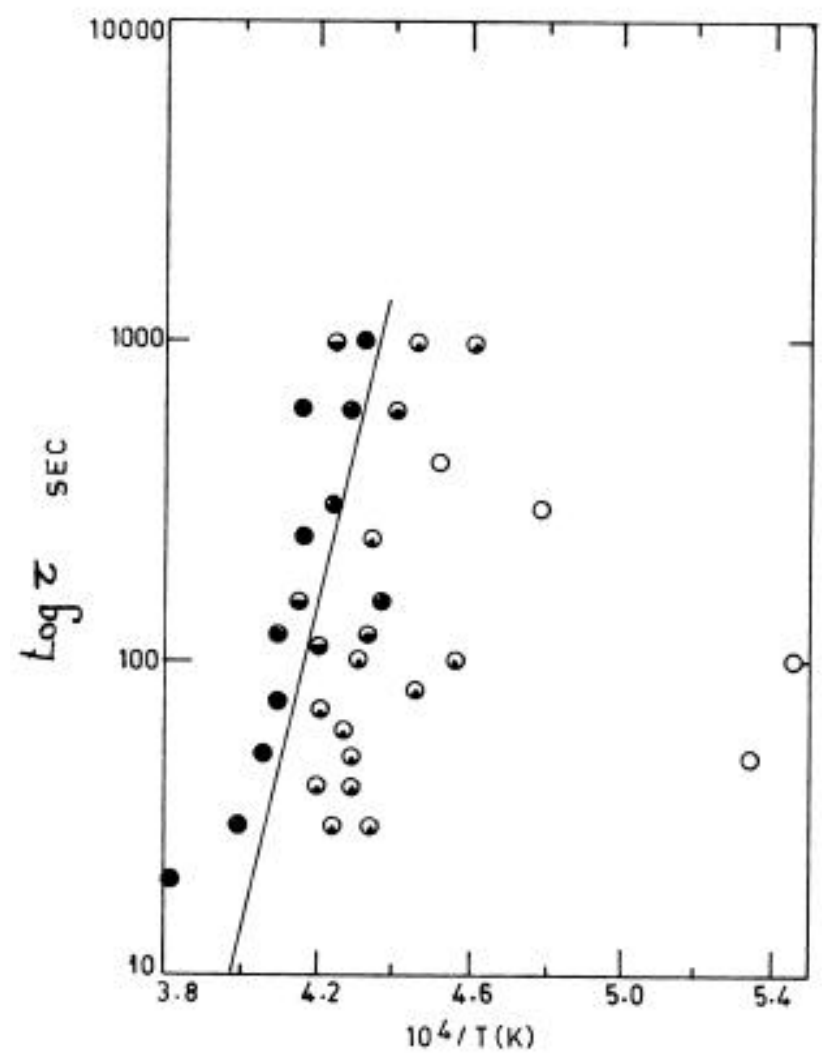

Figure 11. $\log \tau$ vs $10^{4} \mathrm{~T} / \mathrm{K}$ plot of diamond graphitization at 20 kbar. 
eventually successful and diamond synthesis from graphite became possible through the use of solvent catalysts in high pressure high temperature apparatus (Bundy et al 1955).

\section{$7 \cdot 2 \mathrm{GeO}_{2}$}

Germanium oxide occurs in the low temperature rutile form and the high temperature $\alpha$-quartz form. Although the transformation of rutile $\rightarrow \alpha$-quartz is reconstructive and interface controlled transformation and does not come under the purview of this review it is interesting to see a work on kinetics with rigorous analysis of the data. The work by Brar and Schloessin (1981), also summarizes the earlier work by Zeto and Roy (1969). The high pressure $\mathrm{X}$-ray diffraction experiments were done using boron nitride pressure transmitter, molybdenum furnace in

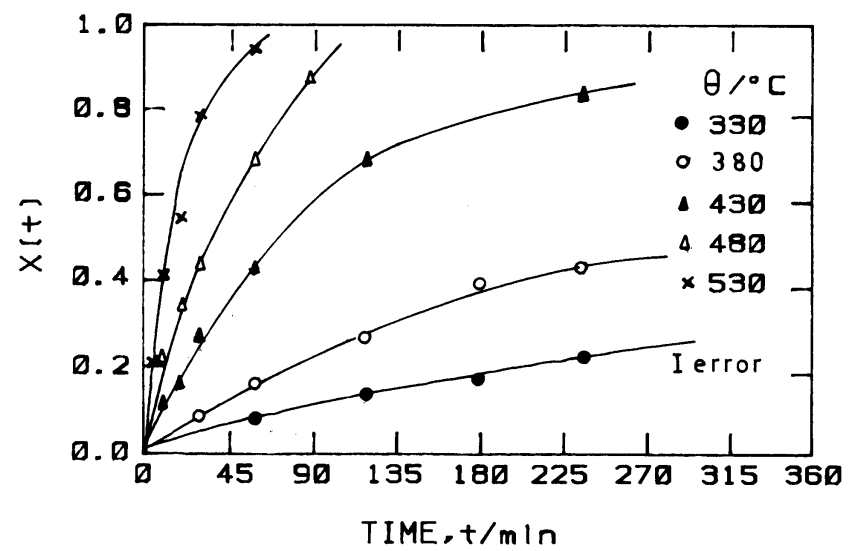

Figure 12. Transformed mass fraction $x(t)$ of rutile $\mathrm{GeO}_{2}$ as a function of time $t$, at a pressure of $2 \mathrm{GPa}$ and at various temperatures, $\theta$ (Brar and Scloessin 1981). opposed anvil configuration. The transformed mass $x(t)$ is determined from the growing Bragg peak intensity ratios. The transformation kinetics is described by a rate equation for the transformed mass or volume fraction $x(t)$, which has the form

$$
x(t)=1-\exp \left(-k t^{n}\right)
$$

where $t$ is the time, $k$ the rate constant, and $n$ is an exponent which characterizes the transformation process, particularly the nucleation and growth topology. Figure 12 gives the transformed mass fraction $x(t)$ of rutile $\mathrm{GeO}_{2}$ as a function of time $t$ at a pressure of $2 \mathrm{GPa}$ and at various temperatures. Figures $13 \mathrm{a}$ and $\mathrm{b}$ give the plot of $\ln \{\ln [1 /(1-x)]\}$ vs $\ln t$ obtained by using the data from figure 12 . The slopes of these plots determine the value of the exponent $n$, which for a very short initial stage of the transformation will be around 4 , in accordance with the formation of the nuclei at the available particle and grain boundary interfaces. These sites are so rapidly exhausted that experimental runs with a total duration of $\ll 5 \mathrm{~min}$ would be required to measure the value of $n$ in this stage. Since in this report the high pressure experiments were run for at best $5 \mathrm{~min}$, most of the observed transformation occurred after the growth of existing nuclei. A value of $n$ around 1 signifies the surface nucleation is homogeneous, and that growth is isotropic as well as near constant. The intercepts of the plots determine the values of the rate constant $k$. The data has been analysed using the Cahn theory of solid state reactions for growth controlled stage proceeding from surface and grain boundary nucleations. The values of the exponent $n$ and the rate constant $k$ obtained from these analyses are summarized in table 6 . The conclusions drawn from the table were (i) the rate constant $k$ increases with increasing temperature at con-
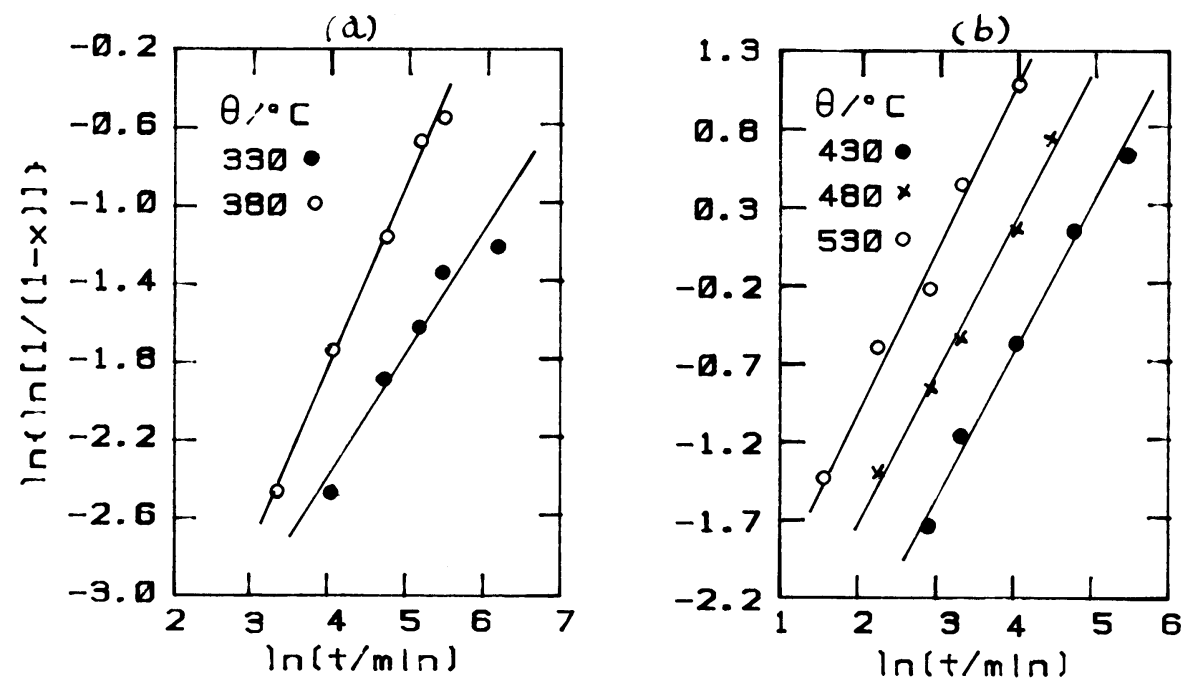

Figure 13. (a) and (b). Plots of $\ln \{\ln [1 /(1-x)]\}$ vs $\ln (t)$ obtained by using the data from figure 12 (Brar and Scloessin 1981). 
stant pressure; and (ii) the value of the exponent $n$ calculated from this work is close to 1 and this characteristic of a grain boundary nucleated solid-solid transformation. In the case of the data of Zeto and Roy (1969) it turns out that the value of $n$ are substantially less than one. This departure of the value of $n$ from one is also remarked upon by Zeto and Roy in their paper. They were not able to establish any relationship of their data with any of the existing kinetic equations. It is possible that the sample geometry dictated by the opposed anvil apparatus imposes boundary conditions for nucleation and growth which are not entirely compatible with that of the Cahn's theory.

\subsection{Ti and $Y b$}

The results obtained by Singh $(1983,1985)$ on the kinetics of pressure induced transformation in titanium and fcc-bcc transformation in ytterbium are summarized in this section. The change in electrical resistance of the specimen is used to monitor the progress of the transformation. Singh et al (1983a) have developed a method for rapidly loading an opposed anvil set up for studying the kinetics under isobaric conditions.

The progress of the transformation was followed by monitoring the resistance of the specimen. The fraction of the high pressure phase as function of time was calculated from the specimen resistance vs time data. The following features were observed in both $\mathrm{Ti}$ and $\mathrm{Yb}$.

(I) The transformation started with a definite incubation period. Results indicate that observed period is the inherent property of the transformation.

(II) The transformations are isobaric-isothermal type.

(III) The kinetics data satisfied Avrami equation with $n=3 \pm 1$ at $5 \mathrm{GPa}$ and decreased to $1 \pm 0 \cdot 2$ at $9 \mathrm{GPa}$ for Ti. For fcc-bcc transitions in $\mathrm{Yb}, n=1 \cdot 1 \pm 0 \cdot 2 \mathrm{GPa}$ and decreased to $n=0 \cdot 3 \pm 0 \cdot 1$ at $4.8 \mathrm{GPa}$. Although the lowest values of $n=1$ as per the theoretical models here $n<1$.

Table 6. Comparison of parameters $n$ and $k$ for $\alpha$-quartz $\rightarrow$ rutile transformation in $\mathrm{GeO}_{2}$ between Merkau and Holzapfel (1986) and Kruger et al (1990).

\begin{tabular}{lcccc}
\hline & $P(\mathrm{GPa})$ & $\theta^{\circ} \mathrm{C}$ & $\mathrm{k} / \mathrm{s}^{-1}$ & $n$ \\
\hline Merkau and Holzapfel & $2 \cdot 0 \pm 0 \cdot 1$ & 330 & $5 \cdot 06 \times 10^{-5}$ & $0 \cdot 64$ \\
$(1986)$ & $2 \cdot 0 \pm 0 \cdot 1$ & 380 & $7 \cdot 03 \times 10^{-5}$ & $0 \cdot 95$ \\
& $2 \cdot 0 \pm 0 \cdot 1$ & 430 & $3.05 \times 10^{-4}$ & $0 \cdot 91$ \\
& $2 \cdot 0 \pm 0 \cdot 1$ & 480 & $4 \cdot 72 \times 10^{-4}$ & $0 \cdot 97$ \\
& $2 \cdot 0 \pm 0 \cdot 1$ & 530 & $1 \cdot 05 \times 10^{-3}$ & $0 \cdot 96$ \\
Kruger et al (1990) & $2 \cdot 04$ & 430 & $4.71 \times 10^{-3}$ & $0 \cdot 41$ \\
& $2 \cdot 04$ & 480 & $2.20 \times 10^{-2}$ & $0 \cdot 33$ \\
& $2 \cdot 04$ & 540 & $2 \cdot 73 \times 10^{-1}$ & $0 \cdot 14$ \\
& $2 \cdot 04$ & 600 & $6 \cdot 02 \times 10^{-1}$ & $0 \cdot 11$ \\
\hline
\end{tabular}

The activation free energy for the combined process of nucleation and growth is related to the time required for nearly $63 \%$ transformation

$$
\ln (p)=b_{0} \Delta G^{*}(p) / R T
$$

where $b$ is a constant, $\Delta G^{*}(p)$ the pressure dependent activation free energy and $R$ the gas constant, $\Delta G^{*}(p)$ can be calculated if $\tau(p)$ is known. Value of $b$ is estimated. Figure 14 shows pressure dependence of $\tau$. The $\ln \tau$ vs $p$ data is linear for $\mathrm{Yb}$ but shows small curvature in $\mathrm{Ti}$.

The experimentally obtained values of activation free energies are significantly lower than the activation energy for self diffusion. This suggests that diffusion process is not involved in these transformations. In diffusion controlled transformations the activation energy is expected to increase or remains relatively unaltered when pressure in increased. The fact that the activation free energy decreases with increase in pressure in the present case also suggested the absence of any diffusion process in the transformation.

\section{$7.4 \quad B i(I)-(I I)$}

Earlier experiments on bismuth under hydrostatic pressure suggests that $\mathrm{Bi}(\mathrm{I})-(\mathrm{II})$ transition is isobaric in that it runs to completion when pressure is held constant. The time dependence behaviour of the transition indicates that the transition progresses via the step of nucleation and growth. The time for completion of the transition under hydrostatic pressure can range from a few seconds for single crystal specimens to several hundred seconds for polycrystalline specimens. Much faster transition kinetics are observed if a solid pressure transmitting medium is

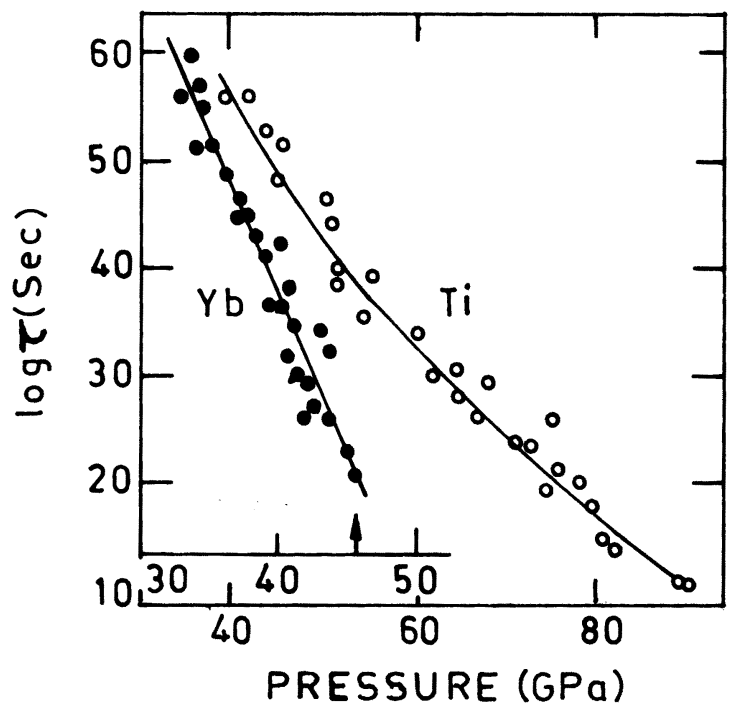

Figure 14. Pressure dependence of $\tau$ for titanium and ytterbium (Singh 1983, 1985). 
used. Singh (1990), in his work reports the kinetics of $\mathrm{Bi}(\mathrm{I})$-(II) transition under constant pressurization rate which can be varied between 0.03 and $0.1 \mathrm{GPa} / \mu \mathrm{s}$. Figure 15 shows the slope of the pressure pulse to be a typical impact experiment (Singh 1989). The change in the resistance of $\mathrm{Bi}$ specimen under $\alpha=0.074 \mathrm{GPa} / \mu$ s at intervals of $1 \mu \mathrm{s}$ is shown in figure 16 . In the region $A-B$ the specimen is in $\mathrm{Bi}(\mathrm{I})$ phase and the resistance increases with time occurs because the pressure increases with time. The drop in resistance at $B$ is associated with the $\mathrm{Bi}(\mathrm{I})-(\mathrm{II})$ transition. The resistance increase beyond $C$ marks $\mathrm{Bi}(\mathrm{II})-$ (III) transition. The magnitudes of the resistances at $B$ and $C$ relative to the initial resistances compare very well with the corresponding numbers obtained in the static experiments with talc as the pressure transmitting medium. The fraction of $\mathrm{Bi}$ (II) formed as a function of time (figure 17) was calculated from the resistance data (Singh et al 1983b; Singh 1985). The time taken for $95 \%$ transformation decreases with increasing pressurization rate and reaches a value of $8 \mu \mathrm{sec}$ at $\alpha=0.1 \mathrm{GPa} / \mu$ s as shown in figure 18 .

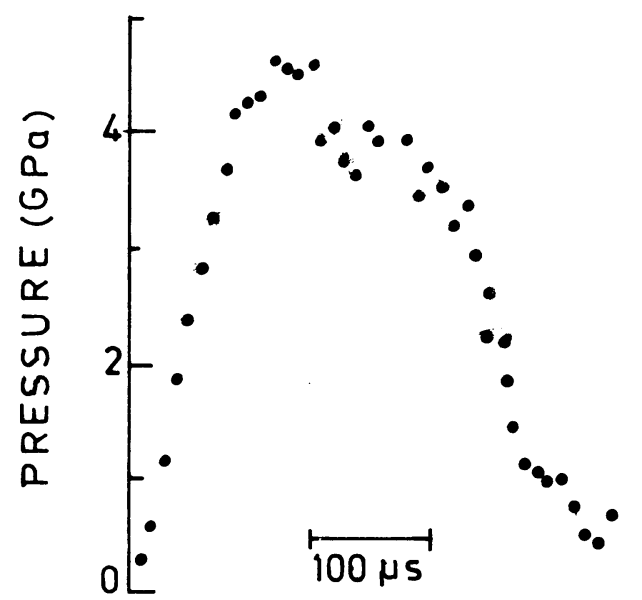

Figure 15. Shape of the pressure pulse in a typical impact experiment (Singh 1990).

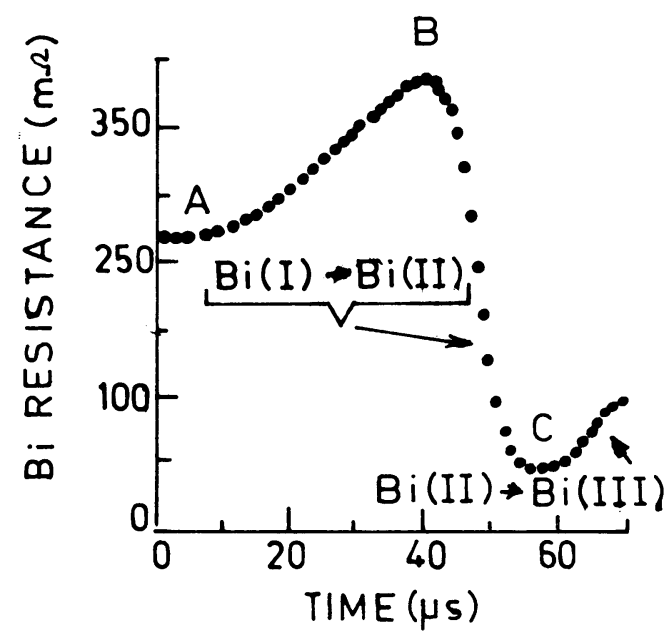

Figure 16. Change in $\mathrm{Bi}$ resistance with time during impact (Singh 1990).

\subsection{Amorphization under pressure}

Recently experimental evidence for pressure induced amorphization has piled up (Meade and Jeanloz 1990; Serghiou et al 1992; Sikka 1992; Sharma and Sikka 1996; Arora 2000). The recent paper by Arora (2000) gives an idea of the number of systems known to show pressure induced amorphization (PIA) and, more interestingly pressure induced decomposition (PID). This paper also points out the central role played by kinetics in these phenomena. Table 7 is reproduced from Sikka (1992) which gives the list of some of the materials showing pressure induced amorphization. The material is amorphized under pressure due to the structural frustration in reaching the dense packed high pressure equilibrium phase. This is the

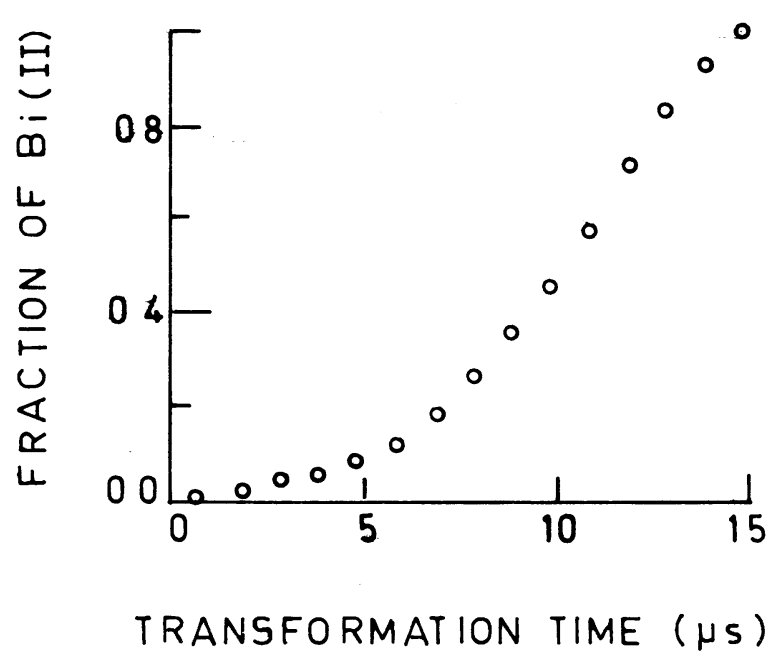

Figure 17. Fraction of $\mathrm{Bi}(\mathrm{II})$ phase formed during impact (Singh 1990).

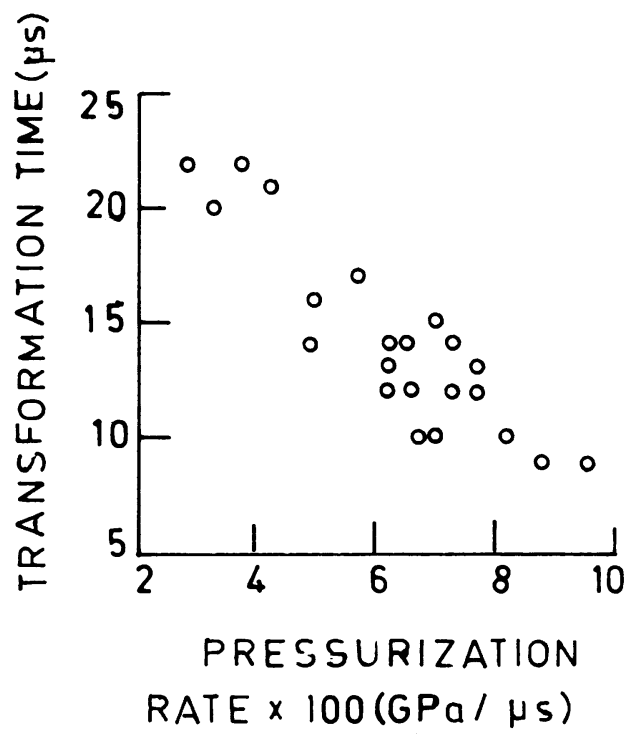

Figure 18. Time for $95 \%$ transformation as a function of pressurization rate (Singh 1990). 
Table 7. List of substances undergoing $c \rightarrow a$ transition under pressure (Sikka 1991).

(i) On decompression: $\mathrm{NaAlSi}_{2} \mathrm{O}_{6}$, stishovite, $\mathrm{CaSiO}_{3}, \mathrm{Al}-\mathrm{Ge}$, $\mathrm{Cd}-\mathrm{Sb}$, and $\mathrm{Zn}-\mathrm{Sb}$, ice-VIII, GaP, GaAs

(ii) On compression: ice- $I_{\mathrm{h}}, \mathrm{SnI}_{4}, \mathrm{TCNE}, \mathrm{LiKSO}_{4}, \mathrm{SiO}_{2}$ (quartz, coesite), $\mathrm{CaAl}_{2} \mathrm{Si}_{2} \mathrm{O}_{8}, \quad \mathrm{Al}_{6} \mathrm{Li}_{3} \mathrm{Cu}$ (quasicrystal), $\mathrm{GeO}_{2}$, $\mathrm{Ca}(\mathrm{OH})_{2}, \quad \mathrm{Fe}_{2} \mathrm{SiO}_{4}, \quad \mathrm{AlPO}_{4}, \quad \mathrm{~Pb}_{2} \mathrm{Nb}_{2} \mathrm{O}, \quad \mathrm{GeI}_{4}, \quad \mathrm{NH}_{4} \mathrm{VO}_{3}$, recorsinol, poly-4-methyl-pentane-1, $\mathrm{Bi}_{4} \mathrm{Ge}_{3} \mathrm{O}_{12}, \mathrm{MgSiO}_{4}$

result of unfavourable kinetics and is thermodynamically symbolized by a three-level diagram (Johnson 1986; Sikka 1992; Sikka and Sharma 1992a, b). The three-level diagram is obeyed by several methods, which induce direct $c \rightarrow a$ transition: interdiffusion, radiation damage, mechanical milling etc. According to this, in order to create an amorphous state, a crystalline solid has to be driven into a high energy state $\left(G_{0}\right)$. This free energy of the crystalline state can be lowered either by the formation of a metastable amorphous phase $\left(G_{\mathrm{a}}\right)$ or by the formation of the more energetically favoured crystalline phase $\left(G_{\mathrm{c}}\right)$. However, the kinetics of the system is such that though the transition from $G_{0}$ to $G_{\mathrm{c}}$ brings the system to a lower energy state, the transition from $G_{0}$ to $G_{\mathrm{a}}$ is favoured. In high pressure experiments, such conditions can be created if the solid has a schematic phase diagram shown in figures 19a and b. The kinetic conditions can be met in two ways: (i) by carrying out experiment at low enough temperatures so that the transformation rate for I-II phase change $\left(k T / \Delta G^{*}\right.$, where $\Delta G^{*}$ is the free energy barrier between I and II) is negligible and (ii) by applying pressure rapidly such as in shock experiments so that transition I-II is suppressed.

Sikka and Sharma (1992a, b) have tried to explain the driving mechanism and it is laid out briefly in the following. The explanation is based on a quote from Kitaigordsky (1980). If the shape of the molecules is such that the formation of the long range order leads to a larger percentage of empty space than in the absence of such an order in a close packing arrangement, then no crystal is obtained. For example most of the substances listed in table 7 contain tetrahedral building blocks and occur at ambient conditions in very open structures. Now, application of pressure promotes close packing but one cannot do so with tetrahedra. However, the building blocks may distort and try to change into more favourable shapes for close packing (by coordination change or by dissociation). This may involve bond breaking/bond formation and diffusion of some atoms. The latter, usually, needs more thermal activation. Since this is inhibited at low temperatures of the experiments, further compression may result in steric hinderances and ultimately destabilize the crystal structure.

$\mathrm{X}$-ray diffraction experiments on $\alpha$-quartz (Hazen et al 1989) and molecular dynamics calculations (Somayazulu
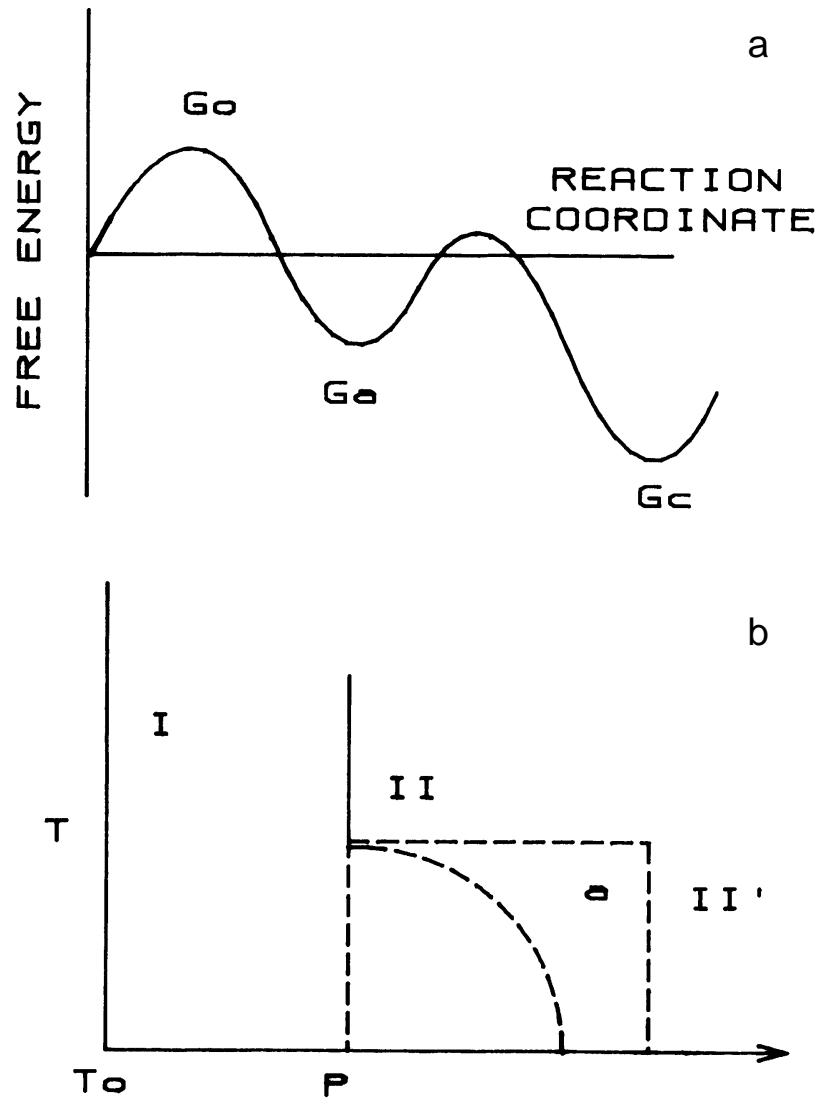

Figure 19. a. The three-level energy diagram for pressure induced $c \rightarrow a$ transition. The kinetic conditions require $(o \rightarrow c) \ll(a \rightarrow c)$ and $(o \rightarrow a) \gg(a \rightarrow c)$ (Sikka 1991) and b. a schematic phase diagram for $c \rightarrow a$ transition under pressure. Phases I and II are the same in some cases. $T_{0}$ is the temperature of the experiment. Dashed lines are kinetic boundaries (Sikka 1991).

et al 1993) fully support the above picture. Recently $\mathrm{C}_{60}$ fullerite has been shown to transform to amorphous carbon near $20 \mathrm{GPa}$ (Snoke et al 1992). The reason for this transformation is again the steric hindrance produced by short intermolecular van der Waals contacts. Experiments on BAs (boron arsenide) have shown that it transforms from zinc blende to an amorphous structure at $125 \mathrm{GPa}$ (Greene et al 1994). Greene et al (1994) have claimed that this is the first evidence that pressure induced amorphization in binary $A B$ type compound, is a kinetically frustrated process.

\section{Conclusions}

At the moment the theoretical models are far from satisfactory in explaining the kinetics of phase transitions. But the experience on phase change kinetics are growing. In kinetics experiments there are, in many cases, some problems with the interpretation of kinetic data with the classical Avrami theory where Avrami exponent is used to 
characterize the mechanism of the phase transition. Especially in the DAC with its small sample volume the change of pressure with time, as well as preparation conditions and the time resolution, may strongly influence the results of kinetic experiments. Because of the problem of keeping the pressure constant during the phase transformation, it is useful to modify the classical Avrami theory by including the time dependence of the thermodynamic potential. The model description thus obtained comprises the influence of the volume change of the sample, as well as the relaxation of the soft gasket materials $(\mathrm{Cu}, \mathrm{Fe}-\mathrm{Ni}, \mathrm{Ni})$ during the transformation process. Furthermore, the experimental cluster distributions and nucleation rates support theoretical models and simulations of the microstructure (Orgzall and Lorenz 1988).

The $B 1-B 2$ phase transition in alkali halides was intensively studied because of their simple coordination structure and low transition pressures $(<3 \mathrm{GPa})$. Traditionally the mechanism of solid-solid bulk transformation in alkali halides was investigated: the martensitic transition and the hysteresis of the retransformation in alkali halides were estimated by means of the volume change with a water free preparation (1:1 mixture of normal pentane and isopentane) (Lacam and Peyroneau 1976). Qualitative measurements of the transformation behaviour in $\mathrm{KCl}$ with EDXD are for example used to determine the equilibrium pressure (Hamaya and Akimoto 1981). Therefore the transition rates during the transformation and retransformation processes are measured. The kinetic investigations in lanthanides (Merkau and Holzapfel 1986; Kruger et al 1990) by EDXD or the phase transition in ytterbium by measurement of electrical conductivity (Singh 1985) led to an Avrami exponent near 1 which is often discussed within the Avrami-Cahn theory as a growth process with a heterogeneous nucleation at grain boundaries. An Avrami exponent lower than 1 could not be explained within the framework of the classical theory. The time resolution of the kinetic data can strongly affect the interpretation of the experimental results and a time resolution of some millisecond is necessary to investigate the first stage of a $s-s$ phase transformation in $\mathrm{KCl}$. However, these experiments cannot unambiguously show whether a transition is a nucleation or a growth process or martensitic one. At the first stage the nucleation and growth of the Avrami theory applies as a good approximation of the real process, but in most of the kinetic experiments it is impossible to investigate the early transformation period. A subject of increasing theoretical interest in recent years has been the similarities of the real structure properties in solids, which can strongly influence the recrystallization processes. The influence of the grain boundaries and lattice defects during nucleation and growth process was investigated by computer simulation of thermodynamic systems. The work in this field is a direct continuation of the classical theory (Srolovitz et al 1986).
The field of pressure induced amorphization has rekindled the interests of physicists in the kinetics of such phase transitions. The literature survey reveals that the experimental evidences throw more questions than answers. There are contradicting reports as to the occurrence of a reversible $c \rightarrow c$ phase transitions just prior to amorphization. A general picture is yet to emerge. Questions like do the displacive transitions show amorphization? If the phase transitions are 'mixed' in order, what component of diffusion controlled mechanism and its effect on the kinetics of the transition, arise? The reconstructive type of transitions generally occur in covalently bonded materials. However, these display some percentage of ionicity and the correlation between the ionicity and mechanism and kinetics of the phase transition will be very interesting and valuable study. These are some of the interesting questions which may be deciding the future directions in this field.

High pressure synthesis is an area with a long history, and new opportunities have been opened by advances in high pressure technology over the past decade. Using diamond anvil cells, it is now possible to compress materials to unprecedented pressures (in excess of $4 \times 10^{6}$ atom or $400 \mathrm{GPa}, 1 \mathrm{GPa} \approx 10,000 \mathrm{~atm}$ ) while simultaneously laser heating (LHDAC) them to temperatures as high as $5000 \mathrm{~K}$ (Hemley et al 1987). Elevated temperatures facilitate the synthesis and processing of new materials at ultra high pressures. Chemical reactions are typically found to proceed rapidly at temperatures exceeding $1000 \mathrm{~K}$, and stress variations across the sample are reduced or elevated during thermal annealing. Very few laboratories have so far explored the pressure-temperatures regime above 30$50 \mathrm{GPa}$ and 1000-2000 K and hence only a limited number of examples exist (Parker et al 1996; Yoo et al 1997). Efforts are on at IGCAR to set up the LHDAC instrumentation in the coming years (Sahu 1998). It is proposed to attempt synthesis of novel III-V compounds like B-Sb, nitrides of carbon and boron with supposed superhard properties and new forms of carbon networks.

\section{Acknowledgement}

We thank our colleagues Dr Mohammad Yousuf, Dr P Ch Sahu, and Dr N Subramanian for discussions and valuable suggestions.

\section{References}

Arora A K 2000 Solid State Commun. 115665

Avrami M 1939 J. Chem. Phys. 71103

Avrami M 1940 J. Chem. Phys. 8212

Avrami M 1941 J. Chem. Phys. 9177

Bassett W A et al 1996 Mineral spectroscopy: A tribute to Roger G Burns (eds) M D Dyar, C McCammon and M W Schaefer (The Geochemical Society Special Publication) p. 261 
Becker R and Doring W 1935 Ann. Phys. 24719

Brar N S and Scloessin H H 1981 High Temp. High Press. 13 313

Bridgman P W 1916 Proc. Am. Acad. Arts Sci. 5257

Bridgman P W 1940 Proc. Am. Acad. Arts Sci. 741

Bridgman P W 1947 J. Chem. Phys. 1592

Brown J M and McQueen R G 1986 J. Geophys. Res. 917485

Bundy F P, Hall H T, Strong H M and Wentorf R J Jr 1955 Nature 17651

Bundy F P 1969 Reactivity of solids, Proc. sixth int. symp. reactivity of solids (eds) J M Mitchell, R C DeVries, R W Roberts and P Cannon (New York: Wiley-Interscience) p. 817

Burke J E and Turnbull D 1952 Prog. metal phys. (eds) B Chalmers and R King (London: Pergamon) Vol. 3 p. 220

Cahn J W 1956 Acta Metall. 4449

Chaplot S L and Sikka S K 1992 Recent trends in high pressure research, Proc. of the XIII AIRAPT conference on high pressure science and technology (ed.) A K Singh (New Delhi: Oxford \& IBH) p. 259

Chaplot S L and Sikka S K 1993 Phys. Rev. B47 5710

Christian J W 1975 The theory of transformation in metals and alloys (Oxford: Pergamon Press) 2 edn

Chvoj Z and Kozisek Z 1991 Kinetic phase diagrams, studies in modern thermodynamics (eds) Z Chvoj, J Sestak and A Triskta (Amsterdam: Elsevier) Ch. 5, p. 145

Cohen M, Olsen G B and Clapp P C 1979 Proc. ICOMAT'79 (Cambridge: MIT-MA) p. 1

Dachlli F and Roy R 1961 Reactivity of solids (ed.) J Boer de (Amsterdam: Elsevier) p. 502

Dassler R 1988 High Temp. High Press. 20661

Dassler R 1990 High Temp. High Press. 22599

Dassler R and Riedel M 1992 High Press. Res. 9120

Delaey L 1991 Phase transformations in materials, materials science and technology (eds) R W Cahn, P Haasen and E J Kramer (New York: VCH) Vol. 5

Gomon G O, Rovsha V S and Shemanin V I 1974 Sov. Phys. Dokl. 18715

Goto Y 1964 Jap. J. Appl. Phys. 3739

Greene R G, Luo H, Ruoff A L, Trail S S and Di Salvo F J Jr 1994 Phys. Rev. Lett. 732476

Gupta S C 1992 Shock compression of condensed matter-1991 (eds) S C Schmidt, R D Dick, J W Forbes and D G Tasker (Amsterdam: Elsevier) p. 157

Gupta S C and Chidambaram R 1994 High Press. Res. 1251

Gupta S C, Daswani J M, Sikka S K and Chidambaram R 1993 Curr. Sci. 65399

Hamaya N and Akimoto S 1981 High Temp. High Press. 13347

Hamaya N, Yamada Y, Axe J D, Belanger D P and Shapiro S M 1986 Phys. Rev. B33 7770

Hazen R M, Finger L W, Hemley R J and Mao H K 1989 Solid State Commun. 7272

Hemley R J and Mao H K 1997 Encyclopedia of applied physics (New York: VCH) Vol. 18, p. 555

Hemley R J and Ashcroft N W 1998 Physics Today 26

Hemley R J, Bell P M and Mao H K 1987 Science 237605

Hirsh K R and Holzapfel W B 1983 J. Phys. E: Sci. Instrum. 16 412

Hixon R S, Boness D A, Shaner J W and Moriarty J A 1989 Phys. Rev. Lett. 62637
Hochheimer H D and Etters R D 1991 Frontiers in high pressure research NATO ASI series, Series B: Physics (New York: Plenum Press) Vol. 286

Hollman J H 1950 Thermodynamics in metallurgy (Ohio: Am. Soc. Met.) p. 161

Huang W L 1996 Org. Geochem. 2495

Huang W L and Otter G A 1998 Org. Geochem. 291119

Jeanloz R 1987 J. Geophys. Res. 9210352

Jayaraman A 1983 Rev. Mod. Phys. 5565

Jayaraman A 1986 Rev. Sci. Instrum. 571013

Jayaraman A 1990 Met. Mater. \& Process. 21

Johnson W L 1986 Prog. Mater. Sci. 3081

Johnson W A and Mehl R F 1939 Trans. Am. Inst. Mining Met. Engrs. 135416

Klement W Jr and Jayaraman A 1967 Prog. in solid state chem. (ed.) H Reiss (London: Pergamon) Vol. 3, p. 289

Kitaigordsky A I 1980 Order and disorder in the world of atoms (Moscow: Mir)

Kruger T, Merkau B, Grosshans W A and Holzapfel W B 1990 High Press. Res. 2193

Lacam A and Peyroneau J 1976 High Temp. High Press. 8628

Landauer R 1952 J. Appl. Phys. 23779

Lorenz B 1992 High Press. Res. 9107

Lorenz B and Lorenz H 1989 Semicond. Sci. Technol. 4288

Lorenz B and Orgzall I 1991 Frontiers in high pressure research (eds) H D Hochheimer and R D Etters (New York: Plenum) p. 243

Lorenz H, Lorenz B, Kuhne U and Hohlfeld Ch 1988 J. Mater. Sci. 233254

Mao H K, Wu Y, Shu J F, Hemley R J and Cox D E 1990 Solid State Commun. $\mathbf{7 4} 1027$

Meade C and Jeanloz R 1990 Geophys. Res. Lett. 171157

Merkau B and Holzapfel W B 1986 Physica B139\&140 251

Miller P J, Block S and Piermarini G J 1991 Combustion \& Flame 83174

Onodera A 1972 Rev. Phys. Chem. Jap. 411

Orgzall I and Lorenz B 1988 Acta Metall. 36627

Parker L J, Atou T and Badding J V 1996 Science 27395

Pistorius C W F T 1976 Prog. in solid state chem. (ed.) H Reiss (London: Pergamon) Vol. 2, p. 1

Rajan K G, Sahu P Ch and Yousuf M 1986 Indian J. Pure \& Appl. Phys. 27461

Rao C N R and Rao K J 1967 Prog. in solid state chem. (ed.) H Reiss (London: Pergamon Press) Vol. 4

Rao C N R and Rao K J 1978 Phase transitions in solids (New York: McGraw Hill)

Riedel M and Dassler R 1990a High Press. Res. 4333

Riedel M and Dassler R 1990b J. Cryst. Growth 106695

Roy R 1969 Reactivity of solids (eds) J M Mitchell, R C Devries, R W Roberts and P Cannon (New York: WileyInterscience) p. 777

Roy R 1973 Phase transitions (eds) H K Heinisch, R Roy and L E Cross (Oxford: Pergamon Press)

Ruoff A L 1991 Phase transformation in materials, materials science and technology (eds) R W Cahn, P Haasen and E J Kramer (New York: VCH) Vol. 5

Sahu P Ch 1994 Electrical resistivity and X-ray diffraction studies of some early actinide systems under high pressure and temperature, Ph.D. Thesis, University of Madras, Madras Sahu P Ch 1998 Solid State Phys. (India) 4163 
Serghiou G C, Winters R R and Hammack W S 1992 Phys. Rev. Lett. 683311

Sharma S M and Sikka S K 1996 Prog. Mater. Sci. 401

Shekar N V C 1997 Investigation of some intermetallic compounds under high pressure using a diamond anvil cell, Ph.D. Thesis, University of Madras, Madras

Sikka S K 1991 Recent trends in high pressure research (ed.)

A K Singh (New Delhi: Oxford \& IBH) p. 254

Sikka S K 1992 Met. Mater. Process. 3303

Sikka S K and Sharma S M 1992a Curr. Sci. 63317

Sikka S K and Sharma S M 1992b High Press. Res. 10675

Singh A K 1983 Bull. Mater. Sci. 5219

Singh A K 1985 Mater. Sci. Forum 3291

Singh A K 1989 Rev. Sci. Instrum. 60253

Singh A K 1990 High Press. Res. 4336

Singh A K 1992 Recent trends in high pressure research, Proc. of the XIII AIRAPT (New Delhi: Oxford \& IBH)

Singh A K, Divakar C and Mohan M 1983a Rev. Sci. Instrum. 541407

Singh A K, Mohan M and Divakar C 1983b J. Appl. Phys. 54 5721

Skriver H L 1985 Phys. Rev. B31 1909

Somayazulu M S, Sharma S M, Garg N, Chaplot S L and Sikka S K 1993 J. Phys. D: Cond. Matter 56345

Snoke D W, Raptis Y S and Syassen K 1992 Phys. Rev. B45 14419
Spain I L and Paauwe J 1977 High pressure technology (New York: Marcel Dekker) Vols I and II

Srolovitz D J, Grest G S and Anderson M P 1986 Acta Metall. 341833

Sung C M 1979 High pressure science and technology (eds) K D Timmerhaus and M S Barber (New York: Plenum) Vol. 2, p. 31

Tse J S and Khy D D 1991 Phys. Rev. Lett. 673551

Turnbull D 1950 J. Chem. Phys. 18198

Turnbull D 1952 J. Chem. Phys. 20411

Turnbull D 1956 Solid state phys. (eds) F Seitz and D Turnbull (New York: Academic Press) Vol. 3, p. 225

Vohra Y K, Brister K E, Wiess S J, Duclos S J and Ruoff A L 1986 Science 2311136

Volmer M and Weber A 1926 Z. Phys. Chem. 119277

Welber B 1976 Rev. Sci. Instrum. 48395

Yoo C S, Akella J, Cynn H and Nicol M 1997 Phys. Rev. B56 140

Yousuf M 1985 Pressure as a parameter in the study of electrical and defect behaviour of metals and alloys, Ph.D. Thesis, Indian Institute of Science, Bangalore

Yousuf M 1998 Semiconductors and semimetals (eds) T Suski and W Paul (San Deigi: Academic Press) Vol. 55, Ch. 7, p. 381

Zeto R J and Roy R 1969 Reactivity of solids (eds) J M Mitchell, R C Devries, R W Roberts and P Cannon (New York: Wiley Interscience) p. 777 